\title{
AGNES: a new electroanalytical technique for measuring free
}

\section{metal ion concentration.}

\author{
J. Galceran ${ }^{* a}$,E. Companys ${ }^{\mathrm{a}}$, J. Puy ${ }^{\mathrm{a}}$, J. Cecilia $^{\mathrm{b}}$ and J.L. Garces ${ }^{\mathrm{c}}$
}

${ }^{\mathrm{a}}$ Departament de Química. ${ }^{\mathrm{b}}$ Departament de Matemàtica. Universitat de Lleida, Rovira

Roure 191, 25198 Lleida, Spain ${ }^{\mathrm{c}}$ Departament de Química Física, Universitat de

Barcelona, Martí i Franqués 1, 08028 Barcelona, Spain * corresponding author

\section{Abstract}

AGNES (Absence of Gradients and Nernstian Equilibrium Stripping) is a stripping technique consisting of two conceptual steps: i) application of a potential program (e.g. a step at a fixed potential) generating a known concentration gain between the outer and inner concentrations of the metal at the mercury electrode surface together with null gradients of the concentration profiles (inside and outside the mercury electrode) and ii) determination of the concentration of reduced metal inside the amalgam in a stripping step. In the present implementation, the stripping step under diffusion limited conditions leads to a measured current just proportional to the free metal ion concentration. In this article we present the basic principles of the technique, analytical expressions for a simplified model of its voltammetric implementation and a numerical study for a more 
refined model together with preliminary experimental results in the system $\mathrm{Cd}(\mathrm{II}) /$ nitrilotriacetic acid showing how this technique can be used as an alternative to other techniques (such as Ion Selective Electrodes) in order to determine free metal activities or concentrations in the presence of complex mixtures avoiding complications such as electrodic adsorption or complexation kinetics.

Keywords: stripping, electroanalysis, sensor, amalgam electrodes, trace metal, speciation

\section{Introduction}

Gaining access to the free concentration of a variety of metal ions such as $\mathrm{Cd}, \mathrm{Zn}, \mathrm{Cu}$ or $\mathrm{Pb}$ is of great interest in numerous fields, amongst which we highlight speciation [1] or biouptake [2] studies. In the former field, the knowledge of the free metal ion concentration together with the total metal and ligand concentrations allows the plotting of the binding curve, a starting point for accurate descriptions of the complexation process in terms of the average equilibrium function [1,3], affinity spectra [4,5], complexation isotherms [6], etc. The interest in the latter field is apparent, for instance, considering how the Free Ion Activity Model (FIAM) [7,8] successfully predicts -for a 
large number of cases- the uptake of metal by organisms as a function of the activity (or concentration) of the ionic species.

Ion Selective Electrodes (ISE) $[9,10]$ are widely used in sensing the free metal concentration, although its practical implementation sometimes lacks stability or reproducibility. Moreover, ISE for some species (such as $\mathrm{Zn}$ ) are not of routine use. So, availability of alternative approaches with conventional equipment could be helpful.

Voltammetric techniques can be seen as complementary tools in the analysis of metal ion solutions with their own advantages and drawbacks. Among the typically claimed advantages, low level of detection is particularly attractive and is usually achieved by stripping techniques [11-13] relying on some pre-concentration step. However, a major problem in voltammetric techniques (such as Anodic Stripping Voltammetry, Stripping Chronopotentiometry or Pulse Polarography) is the complex interpretation of the response functions in order to determine the free metal concentration [14]. So, we aim here at the design of a technique addressed to the sensing of the free ion concentration of amalgamating metals with a less complex interpretative framework. We suggest calling this technique AGNES (Absence of Gradients and Nernstian Equilibrium 
Stripping), because the stripping step is applied on the pre-concentrated analyte after a special situation is reached: there is no concentration gradient of any species and the redox couple has also reached Nernstian equilibrium at the mercury interphase. A kind of precedent of this design are the amalgam electrodes $[15,16]$, but we could highlight 2 main differences: i) AGNES takes advantage of the finite volume effects in small electrodes [17-19] allowing diffusion to generate a flat profile for the amalgamated species at the end of the first conceptual step (i.e., AGNES prepares a sort of amalgam electrode in situ) and ii) the response function to be measured after the prescribed potential program in AGNES is not a potential, but -in this first implementation- the current.

A first aim of this work is to describe the basic concepts of AGNES. This technique could be implemented for a variety of mercury electrodes [20-23], but for simplicity of operation and reproducibility, we describe here a voltammetric implementation with commercially available equipment. Hopefully, the sensing of metal ion concentrations of a variety of metals could be possible without the acquisition of specific electrodes either using standard commercial equipment or even designing cheap implementations 
of the technique here presented. Also for the sake of clarity, we just develop here the simplest potential program (consisting in a constant potential step) for the first conceptual step of AGNES and, for the second step, we just measure the faradaic stripping current (for instance, in another implementation of AGNES, the charge could be also a suitable response function) under diffusion limited conditions.

A second aim of this article is the qualitative analysis of the impact of the technique parameters, while highlighting the need to carefully select them. In the presentation and application of analytical (appendix A) or numerical (appendix B) models, we always refer here to a supposedly spherical electrode of radius $r_{0}$. Extension to a semi-spherical model is straightforward, but not detailed here.

A third aim is to illustrate its application with experimental data. For its well known reversibility, Cd has been chosen. The selected ligand, Nitrilotriacetic acid (NTA), is commonly used in speciation studies with Cd [14,24-28]. Obtaining a very low limit of detection with AGNES is left for further work (probably with another kind of 
electrodes), while here we describe a safe combination of the parameters so that

AGNES requirements clearly apply.

\section{Principles of AGNES}

\subsection{First conceptual step: absence of concentration gradients keeping}

Nernstian equilibrium at the electrode surface

Assume a mercury electrode having a very small volume in contact with a large solution

containing an ion $\mathrm{M}$ (its charge is omitted for the sake of notation simplicity) whose

reduced form, $\mathrm{M}^{\mathrm{o}}$, dissolves into mercury. When a reducing step is applied to this

system, the process

$\mathrm{M}+n \mathrm{e}^{-} \rightarrow \mathrm{M}^{\mathrm{o}}(\mathrm{Hg})$

occurs. If the applied potential $E_{1}$ is just a few milivolts more negative that the standard

potential of the couple and we keep applying the potential step for a long enough time

$t_{1}$, the flux of metal crossing the electrode surface will eventually end, because the Nernstian equilibrium is reached with the free metal concentration at the electrode surface being equal to the bulk concentration $c_{\mathrm{M}}^{*}$. 
Let us designate by $Y$ the ratio of concentrations at each side of the electrode surface

(indicated by superscript 0 ) at any moment of the experiment:

$$
Y \equiv \frac{c_{\mathrm{M}^{0}}^{0}}{c_{\mathrm{M}}^{0}}
$$

When flat profiles have been established at each side of the mercury electrode (see Fig 1a), Nernst equation prescribes

$$
Y=\frac{c_{\mathrm{M}^{0}}^{*}}{c_{\mathrm{M}}^{*}}=\exp \left[-\frac{n F}{R T}\left(E_{1}-E^{\mathbf{o}}\right)\right]
$$

where $E^{\mathrm{o}}$ stands for the formal standard potential of the redox couple and the $c_{\mathrm{M}^{0}}^{*}$ refers to the final homogeneous (flat) concentration value inside the mercury electrode. $Y$ is, thus, the gain in concentration of the metal across the surface, due to its preconcentration in the amalgam following the application of $E_{1}$ and is directly related to the difference $\left(E_{1}-E^{\mathrm{o}}\right)$ once $n$ is fixed. Unless the electron transfer for the couple $\mathrm{M} / \mathrm{M}^{\mathrm{o}}$ is extremely sluggish, the time needed until the Nernstian equilibrium (equation 
(3)) is reached will be much less than the time needed for the condition of no concentration gradients (especially with minielectrodes as those used in this work).

The built up concentration $c_{\mathrm{M}^{0}}^{*}$ depends only on $c_{\mathrm{M}}^{*}$ and is independent of any other characteristic of the medium, such as complexation or electrodic adsorption (we assume the existence of a sufficiently large amount of background electrolyte buffering the ionic strength, so that activities and concentrations are always proportional), because of the Nernstian equilibrium prescribed by eqn. (3).

Notice that in order to have constant-value profiles it is necessary to work with a small volume of mercury (relative to its area) so that the finite volume effects arise and the amount of required $\mathrm{M}^{\mathrm{o}}$ can be supplied within a reasonable time $\left(t_{1}\right)$. The time requirements for the specific case of a spherical electrode is discussed below in section 2.2

We caution that strictly flat concentration profiles of $M$ cannot be achieved if the applied potential also sustains another steady-state or transient electrochemical process 
which generates a non-flat concentration profile of some species interacting with $\mathrm{M}$ along the solution. However, if the non-flat profile of the interacting species has a negligible effect on the concentration profile of M, AGNES could be successfully used.

\subsection{Second conceptual step: determination of the concentration of}

reduced metal in the amalgam

The second step of AGNES is the detection of the metal concentration $c_{\mathrm{M}^{0}}^{*}$ preconcentrated inside the mercury electrode along the first step (see Table 1). So, we apply a re-oxidation potential $E_{2}$ and we reverse process (1) to obtain

$\mathrm{M}^{\mathrm{o}}-n \mathrm{e}^{-} \rightarrow \mathrm{M}$

An outline of the resulting concentration profiles is schematised in Fig 1b). The response function measured in AGNES can be the faradaic current ensuing a second potential step under diffusion limited conditions, which will be experimentally accessible as a difference between the total current and the capacitive current of a blank experiment. 
Under diffusion limited conditions just prescribed with $c_{\mathrm{M}^{0}}^{0}=0$, no phenomenon in the solution (such as complexation, convection or adsorption) can affect the faradaic current. Thus, the $I_{\text {faradaic }}$ cannot be dependent on any characteristics of the solution. In this way AGNES avoids the need of knowing parameters of the medium such as ligand concentrations, kinetic constants, diffusion coefficients, hydrodynamic characteristics, etc.

\subsection{A basic relationship for AGNES: the measured current under}

diffusion limited conditions is proportional to the free metal ion

\section{concentration}

As the measured current is linearly related with $c_{\mathrm{M}^{0}}^{*}$ (due to the linear nature of the diffusion equation for $c_{\mathrm{M}^{0}}$ inside the mercury electrode; see appendix $\mathrm{A}$ for the particular case of a spherical drop), and $c_{\mathrm{M}^{0}}^{*}$ is just $Y c_{\mathrm{M}}^{*}$ according to eqn. (3), it follows that the free metal ion concentration is directly proportional to the measured current, with a factor of proportionality 
$h \equiv \frac{I_{\text {faradaic }}}{c_{\mathrm{M}}^{*}}$

controlled via the applied potential $E_{1}$. This factor $h$ also depends on the geometry and

diffusion properties of the mercury electrode, and is analysed below in Appendix A for the particular case of a spherical drop. However, $h$ can be experimentally determined carrying out current measurements (i.e. calibrations) at known free ion concentration (see section 3) with the same electrode, the same applied potential and the same characteristic times for any electrode geometry (even if it was unknown).

\section{A voltammetric implementation of AGNES}

\subsection{Materials and Methods}

\subsubsection{Reagents and Instrumentation}

Cadmium stock solutions were prepared from $\mathrm{Cd}\left(\mathrm{NO}_{3}\right)_{2} \cdot 4 \mathrm{H}_{2} \mathrm{O}$ (Merck analytical grade) and standardised by means of a complexometric endpoint titration with EDTA

[29]. Potassium nitrate was used as inert supporting electrolyte at $0.1 \mathrm{~mol} \mathrm{~L}^{-1}$ and prepared from solid $\mathrm{KNO}_{3}$ (Merck, analytical grade). $\mathrm{KOH}$ titrisol (Merck) and NTA (Fluka, analytical grade) in the $\mathrm{H}_{3} \mathrm{~L}$ form were also used. 
Ultrapure water (Milli-Q plus 185 System, Millipore) was employed in all the experiments. Purified water-saturated nitrogen $\mathrm{N}_{2}(50)$ (purity $\geq 99.999 \%$ ) was used for deaeration and blanketing of solutions.

Voltammetric measurements were carried out with an Eco Chemie Autolab PGSTAT10 potentiostat attached to a Metrohm 663 VA Stand and to a computer by means of the GPES4 (Eco Chemie) software package. The working electrode was a Metrohm multimode mercury drop electrode, the auxiliary electrode was a glassy carbon electrode and the reference electrode was $\mathrm{Ag}|\mathrm{AgCl}|\left(3 \mathrm{~mol}^{-1}\right) \mathrm{KCl}$, encased in a 0.1 mol L-1 $\mathrm{KNO}_{3}$ jacket.

A cadmium specific electrode (Orion 9448) and a glass combined electrode (Orion 9103) were attached to an Orion Research 720A Ionanalyzer. A glass jacketed cell provided by Metrohm was used in all measurements. The vessel was thermostated at $25.0^{\circ} \mathrm{C}$ 


\subsubsection{Procedure}

Solutions were initially purged with oxygen-free nitrogen, then a nitrogen blanket was

maintained during measurements.

AGNES has been performed here by applying a sequential combination of chronoamperometric methods (i.e. current $v s$. time for an applied potential) to a mercury drop, as detailed in Table 1. It can be easily implemented through the GPES program by defining a project which allows the sequential execution of different procedures, i.e. chronoamperometric methods.

In order to be able to use a relatively short preconcentration time $t_{1}$, we have chosen the smallest drop in our stand. According to the catalogue, a radius around $r_{0}=1.41 \times 10^{-4} \mathrm{~m}$ is obtained.

Different $E_{1}$-values were selected to reach certain preconcentration gains, whereas $E_{2}=0$

V was always chosen for the diffusion limited re-oxidation step. 
In the first step, a well stirred solution is suitable to cut down the required $t_{1}$ value. A magnetic stirrer at a speed of $400 \mathrm{rpm}$ was appropriate (not causing the falling of the drop).

A Cd selective electrode was also introduced in the working solution in order to compare both techniques. The potential of the Cadmium selective electrode was read, with a stability criteria of $0.1 \mathrm{mV} \mathrm{min}^{-1}$, in sequence to the AGNES procedure. The Cd ISE was always calibrated previous to the experiment by adding accurate doses of a cadmium stock solution.

\subsection{How long does it take to reach Nernstian Equilibrium without}

concentration gradients?. Choosing the deposition time $t_{1}$.

To provide a guideline to answer this question, a model for AGNES with a spherical mercury electrode is developed assuming reversible behaviour of the couple, i.e. $c_{\mathrm{M}^{0}}^{0}(t)=Y c_{\mathrm{M}}^{0}(t)$ at any time. Due to the stirring we impose the bulk condition at a finite distance $r_{1}$, i.e. $c_{\mathrm{M}}\left(r_{1}, t\right)=c_{\mathrm{M}}^{*}$ while assuming the medium is stagnant in the region 
$r_{0}<r<r_{1}$. The value of $r_{1}$ can be estimated from the steady-state flux via its expression in terms of an effective diffusion layer shell thickness $\delta$ :

$$
D_{\mathrm{M}}\left(\frac{1}{r_{0}}+\frac{1}{r_{1}-r_{0}}\right)\left(c_{\mathrm{M}}^{*}-c_{\mathrm{M}}^{0}\right)=D_{\mathrm{M}} \frac{c_{\mathrm{M}}^{*}-c_{\mathrm{M}}^{0}}{\delta}
$$

M diffuses towards the surface where, along $t \leq t_{1}$, the metal is "pumped up" due to the gain $Y$ prescribed by the applied potential $E_{1}$. More details -such as the need to take into account the finite volume effect in the diffusion of $\mathrm{M}^{\mathrm{o}}$ within the drop- are given in appendix B. The model requires some parameters such as the value of the diffusion coefficient of $\mathrm{M}^{\mathrm{o}}$ (in our case $\mathrm{Cd}$ in $\mathrm{Hg}$ ) for which a wide range of values have been suggested in the literature [30-35] (from those we have chosen the value $1.60 \times 10^{-9}$ $\mathrm{m}^{2} \mathrm{~s}^{-1}$ as a compromise which also fits our results here), the radius of the spherical drop (we take the catalogue value) and the diffusion layer thickness $\delta$ (whose value, for the first computations of the model, is taken from situations in the literature [36,37] with different drop size and stirring rate but still valid as estimation of the order of magnitude of $\delta$ ). This model allows the plotting of the evolution of the concentration profiles seen in Fig 2. Notice in Fig 2a that a practically flat $c_{\mathrm{M}^{0}}$-profile inside the drop is achieved 
very soon (less than 10s) $[38,39]$ and that, for the used parameters, the assumption of reaching $c_{\mathrm{M}}^{0}=c_{\mathrm{M}}^{*}$ is perfectly reasonable at $t_{1}=1300 \mathrm{~s}$.

Inspection of the profiles seen in Fig 2 suggests a simplified model for the present implementation of AGNES with a reversible couple. The key point is to consider that, at any time along the pre-concentration step $t \leq t_{1}$, the small drop radius renders diffusion very effective: i) ensuring a flat profile inside the drop (i.e. $c_{\mathrm{M}^{0}}\left(r \leq r_{0}, t\right)=c_{\mathrm{M}^{0}}^{0}(t)$ ) [38,39] and ii) ensuring the steady-state diffusion profile outside the drop (i.e the readaptation of the diffusion profile to a steady-state situation is faster than the filling up process due to changes in $\left.c_{\mathrm{M}^{0}}^{0}(t)\right)$. These approximations, where the transients are neglected, seem reasonable when the characteristic times for diffusion in both media are short in comparison with the time under consideration: $t>>r_{0}^{2} / D_{\mathrm{M}^{0}}$ and $t>\left(r_{1}-r_{0}\right)^{2} / D_{\mathrm{M}}$. This model is schematised in Fig 3. We equal the variation with time in the number of moles inside the drop to the product of the flux times the area of the drop 


$$
\frac{d}{d t}\left(\frac{4}{3} \pi r_{0}^{3} c_{\mathrm{M}^{0}}\right)=\frac{D_{\mathrm{M}}\left(c_{\mathrm{M}}^{*}-\frac{c_{\mathrm{M}^{0}}}{Y}\right)}{\delta} 4 \pi r_{0}^{2}
$$

and integrate from $t=0$ to $t=t_{1}$ (i.e. we neglect transient effects) to obtain:

$$
1-\frac{c_{\mathrm{M}^{0}}^{*}}{Y c_{\mathrm{M}}^{*}}=\exp \left(-\frac{3 D_{\mathrm{M}} t_{1}}{\delta Y r_{0}}\right)
$$

The 1.h.s. of the previous expression measures the departure of the final concentration inside the drop from the expected value $Y c_{\mathrm{M}}^{*}$ : if there was no departure, then the 1.h.s. would yield a 0 value. Focussing on the r.h.s. of expression (8), we analyse the impact of the different parameters able to be experimentally controlled:

a) Deposition time. Long $t_{1}$ values favour the approach to equilibrium. Mathematically speaking, equilibrium is never reached once the perturbation has been introduced. However, a reasonable proximity to the equilibrium situation is enough for practical purposes. For instance, one could accept $99 \%$ of proximity to the exact equilibrium values by equating the 1.h.s. of eqn. (8) to 0.01, as used in Fig 4. Too long deposition times would render the technique less useful, so controlled parameters, such as $Y$ (see its 
equivalence with $-\left(E_{1}-E^{o}\right)$ comparing the ordinate axes of Fig 4$)$, have to be carefully chosen.

b) Stirring. Shorter $t_{1}$ could be sufficient for a fixed required departure from equilibrium, if $\delta$ becomes smaller due to the enhancement of the effectiveness of the mass transport. So energetic stirring with a magnetic stirrer is applied up to a few seconds (we have taken here $t_{w}=100 \mathrm{~s}$; see Table 1) before the end of the application of $E_{1}$. In the experiments we have noticed that there is always a small current (of the order of $0.4 \mathrm{nA}$ ) along the waiting period $t_{1}-t_{\mathrm{w}}<t<t_{1}$, probably due to the incomplete exclusion of oxygen (say at a concentration of $2.4 \times 10^{-7} \mathrm{M}$ ). If the reduction of $\mathrm{O}_{2}$ was important enough to create a $\mathrm{pH}$ profile influencing the speciation of the metal, then AGNES would just sense the free metal concentration at the local $\mathrm{pH}$ of the electrode surface. This kind of problem has some parallelism with the use of amalgam electrodes [15] and is common to other voltammetric techniques. From our experimental data, we infer that the contribution of $\mathrm{O}_{2}$ reduction in the blank current at $t_{2}$ of the order of $0.2 \mathrm{~s}$ is almost negligible in comparison with other metal traces, so we speculate that $\mathrm{O}_{2}$ can be less important for the limit of detection with AGNES than with the amalgam electrodes. 
c) Deposition potential. Longer $t_{1}$ should be required for a fixed departure from equilibrium, if $Y$ increases and other parameters (such as $r_{0}$ ) are kept constant. The dramatical impact of changing $Y$ is illustrated in Fig 4, indicating the importance of keeping $Y$ not too large, if we do not want to wait long $t_{1}$ values. The progressive increase of $c_{\mathrm{M}^{0}}$ of the drop up to $Y c_{\mathrm{M}}^{*}$ can be experimentally followed (see Fig 5) if we measure currents at increasing pre-concentration times keeping $Y$ relatively high: for short times, the current measured at a fixed $t_{2}$ increases with $t_{1}$ because the concentration in the amalgam is still far from the desired value $Y c_{\mathrm{M}}^{*}$ (see lower quasiflat profiles in Fig 2a). We can perform a simple extension of the model used to derive expression (8) in order to include the waiting time $t_{\mathrm{w}}$-usually $100 \mathrm{~s}$ - along which the stirring ceases, but we keep applying $E_{1}$ (see Table 1). One can, then, obtain the approximate expression:

$$
\left(\frac{I_{\text {faradaic }}}{c_{\mathrm{M}}^{*}}\right)_{t=t_{1}}=\left(\frac{I_{\text {faradaic }}}{c_{\mathrm{M}}^{*}}\right)_{t=\infty}\left(1-\exp \left[-\frac{3 D_{\mathrm{M}}}{Y r_{0}}\left\{\frac{t_{1}-t_{w}}{\delta}+\frac{t_{w}}{r_{0}}\right\}\right]\right)
$$

for the proportionally factor $h$ (see eqn. (5)) applicable even when the flat profile for $c_{\mathrm{M}}$ (i.e. outside the drop) has not been reached yet. Eqn. (9) reproduces the experimental 
measurements in Fig 5 reasonably well, taking into account that transient effects have been neglected, that this expression corresponds to a perfectly spherical electrode and that $\delta$ is the only adjusted parameter to $2.47 \times 10^{-5} \mathrm{~m}$ which is also consistent with literature values $[36,37,40]$.

d) Drop size. Larger radius $r_{0}$ values require longer $t_{1}$-values for a fixed departure from equilibrium; so, in this work, we have used the smallest drop available with our equipment. As seen in Fig 2 (where the catalogue radius of the experimental drop has been used), the hypotheses leading to eqn. (8) are fully acceptable, provided the parameter values are roughly correct. As seen in Fig 4, the use of microelectrodes (i.e. decreasing $r_{0}$ ) should allow to achieve much larger concentration gains ( $Y$-values) for a fixed $t_{1}$.

We conclude that the selection of $t_{1}$ cannot be done independently of the selection of $Y$, which is linked to the selection of the deposition potential (to be discussed in the next section). After some preliminary experiments (see how larger stirred deposition times $t_{1}$ 
$-t_{\mathrm{w}}$ in Fig 6 have no practical effect on the measured currents) with $Y$ of the order of

100 , we have mostly worked with $t_{1}-t_{\mathrm{W}}=1300 \mathrm{~s}$.

\subsection{Choosing the deposition potential}

The selection of the potential $E_{1}$ must take balance of two opposite interests:

a. On one hand, $E_{1}$ should be as less negative as possible, so as to have a small $Y$ (see eqn. (3)) and, as commented in the previous section, have to wait less time

$t_{1}$ for the equilibration with flat profiles. In any case, the solubility limit of the reduced metal in the amalgam [41] poses an upper limit to the $Y$-value.

b. On the other hand, one could be interested in having a large $Y$, in order to obtain measurable currents even with very low $c_{\mathrm{M}}^{*}$ (see eqn. (5) and take into account that $h$ is proportional to $Y$ ) and sense trace levels of free metal ion.

Since this works aims at exploring the implementation of AGNES principles, we have given priority to work with safe combinations of parameters rather than lowering the limit of detection. In the compromise, the $Y$-value chosen for our experiments with AGNES is around 100 (corresponding to $E_{1}$ around $-0.670 \mathrm{~V}$ ). Results in Fig 6 (with 
$Y=124)$ experimentally confirm that $Y=100$ and $t_{1}-t_{\mathrm{w}}=1300 \mathrm{~s}$ is a safe combination for

AGNES hypotheses to be acceptable, as further increase of $t_{1}-t_{\mathrm{w}}$ does not significantly increase the measured current and a lower $Y$ (such as 100) requires a shorter preconcentration time $t_{1}$. This experimental result is also consistent with the estimation derived from the theoretical Fig 4 (where several assumptions are made and the parameter value $\delta$ cannot be taken as very accurate): for the couple ( $\left.r_{0}=141 \mu \mathrm{m}, Y=100\right)$ the required value read for $t_{1}$ from the figure is around $600 \mathrm{~s}$.

\subsection{Choosing the measuring time $t_{2}$}

Again, there are two opposite interests for the selection of the measuring time $t_{2}$ :

a. Shorter $t_{2}$ will produce larger currents, as follows from the decaying expression (A-6) plotted in Fig 7, and would allow to sense lower values of $c_{\mathrm{M}}^{*}$.

b. On the other hand, too short $t_{2}$ values can be more affected by the capacitive current.

In order to look for a minimum in the impact of the capacitive and metal traces current, we have plotted the ratio between the current in a blank experiment without any metal 
and the total current in Fig 8 . The value $t_{2}=0.2 \mathrm{~s}$ seems especially suitable for our experimental set-up. However, we have recorded several other measuring times $t_{2}$ for each AGNES experiment. We caution that the optimum $t_{2}$ can vary with different experimental set-ups.

Further optimisation of the potential program for the first conceptual step (leading to conditions of absence of gradients and Nernst equilibrium in shorter times), the response function to measure (such as current or charge) in the stripping step and other parameters of AGNES is in progress.

\section{Experimental results}

\subsection{Calibration of AGNES with $\mathrm{Cd}^{2+}$ solution}

One way of checking the proportionality between current and free metal concentration

consists in plotting $I v s . c_{\mathrm{M}}^{*}$. As seen in Fig. 9, with the chosen experimental settings, the linear behaviour holds acceptably well $\left(r^{2}=1.000\right)$. The proportionality factor $h$ in the plot ranges from $0.004 \mathrm{~A} \mathrm{M}^{-1}$ (for $t_{2}=2.5 \mathrm{~s}$ ) to $0.138 \mathrm{~A} \mathrm{M}^{-1}\left(t_{2}=0.2 \mathrm{~s}\right.$ ). 
As each milivolt of uncertainty in $E_{1}$ has an impact around $8 \%$ in the value of $Y$ (and then, in the determination of $c_{\mathrm{M}}^{*}$ ) and the reference electrode may exhibit some drift along different days, we have chosen to perform a Differential Pulse Polarography (DPP) experiment (with the largest drop in order to apply planar electrode expressions and in a relatively concentrated solution of $\mathrm{Cd}$ without complexants) immediately before a series of AGNES executions. Thus, $Y$ can be easily computed from the peak potential $E_{\text {peak }}$ in this DPP experiment without having to rely on the nominal value of the applied potential $E_{1}$. By combining eqn. (3) with the well-known expression for the DPP peak for the planar electrode [42] we obtain

$$
Y=\sqrt{\frac{D_{\mathrm{M}}}{D_{\mathrm{M}^{0}}}} \exp \left[-\frac{n F}{R T}\left(E_{1}-E_{\text {peak }}-\frac{\Delta E}{2}\right)\right]
$$

Conversely, if we want to work with a fixed $Y$, we can perform first a DPP experiment and, with the previous expression, compute the required $E_{1}$-value for AGNES.

Notice that the estimation of $Y$ (for instance, with eqn. (10)) is not strictly necessary for the simplest methodology of calibration and target measurement whenever the potential 
drift can be neglected and we are just interested in the unknown concentration, but it has been performed here in order to obtain a deeper understanding of the fundamentals of the technique.

\subsection{Speciation measurements}

In order to assess for the potential use of AGNES in speciation studies we have worked with the system $\mathrm{Cd}^{2+}$-NTA (nitrilotriacetic acid).

In Fig 10 we have plotted the $h$-values obtained for 3 solutions: one containing no NTA and the other two having different NTA concentrations. The $h$-values have been computed as the total current minus the corresponding blank current (i.e. with the same NTA concentration but no Cd) divided by the free metal concentration predicted by MINTEQ [43]. We observe that the $h$-values recovered by AGNES for the different replicates of the 3 solutions are practically constant.

The following procedure has been applied to determine the free metal concentration at different mixtures of $\mathrm{Cd}^{2+}$-NTA. All experiments share common values of: ionic 
strength, drop size, deposition time $t_{1}$ and deposition potential $E_{1}$ (implying a common $Y$ ). Under these conditions, $h$ only depends on the different measuring times $t_{2}$ and can be determined from a calibration experiment with no NTA, where the Cd concentration can be known from the preparation followed. From the $I_{\text {faradaic }}$ corresponding to each $\mathrm{Cd}^{2+}$-NTA solution, simply division of $I_{\text {faradaic }}$ by $h$ (see eqn (5)) allows the determination of $c_{\mathrm{M}}^{*}$ predicted by AGNES.

Agreement of the free ion concentrations found by ISE and by AGNES is seen in Fig 11, where a high correlation coefficient is found together with a slope close to the expected value of 1 . As seen in Fig 12 values of $\mathrm{pCd}$ predicted by MINTEQ, ISE and AGNES compare satisfactorily, with shorter $t_{2}$ (e.g. $0.2 \mathrm{~s}$ ) being more reliable than longer ones.

An estimation of the limit of detection with parameters $Y=100$ and $t_{1}=1400 \mathrm{~s}$ can be done by computing the expected $\left[\mathrm{Cd}^{2+}\right]$ yielding 3 times the blank current. With this procedure we have found a limit of detection of free Cadmium concentration of $7 \times 10^{-8}$ $\mathrm{M}$ in this system with NTA. Obviously this limit of detection could be lowered (with 
the present drop size) if we used larger $Y$-values (but would require longer preconcentration times $t_{1}$ )

\section{Conclusions}

In the expound implementation of AGNES the faradaic measured current is directly proportional to the free metal ion concentration without complications arising from electrodic adsorption or other phenomena in the medium. Calibration of the (sufficiently small) mercury electrode with a known $c_{\mathrm{M}}^{*}$, allows the determination of an unknown $c_{\mathrm{M}}^{*}$ in a sample (see eqn. (5)) .

AGNES can, then, be a complementary technique to determine free metal ion concentrations even with conventional voltammetric equipment. Special care has to be exercised in choosing balanced settings for the deposition potential and preconcentration time. In this respect, the spherical model developed here provides some guidelines for choosing these parameters and, because of the agreement between observed and expected currents, supports the interpretation of the whole experiment. 
The experimental results with a drop area around $0.25 \times 10^{-6} \mathrm{~m}^{2}$, show that AGNES with the settings $t_{1}=1400 \mathrm{~s}, t_{\mathrm{w}}=100 \mathrm{~s}, E_{1}$ around $90 \mathrm{mV}$ more negative than the $E_{\text {peak }}$ in a DPP experiment with $\Delta E=-50 \mathrm{mV}, E_{2}=0 \mathrm{~V}$ and $t_{2}=0.2 \mathrm{~s}$ successfully senses the free Cadmium concentration in the presence of NTA.

Due to the potentiostatic nature of its first step, the accuracy of AGNES has a similar exponential dependence with the inaccuracy of the applied potential to that exhibited by the ISE or the amalgam electrodes. So, potential drifts of the reference electrode have to be carefully controlled or followed from calibration to the determination of the metal ion in the sample. Another source of inaccuracy in the presented implementation is the reproducibility of the drop size, although -in principle- this can be accounted for via averaging measurements.

The need to wait for relatively long times (e.g. 20 minutes per measurement adopted in this preliminary work to be on the safe side) is perhaps the most serious drawback of the here expound AGNES implementation with the voltammetric stand. This difficulty could be solved using other mercury electrodes such as microelectrodes or thin film 
electrodes or using a more complex potential program for the first conceptual step.

Optimisation of the experimental settings (in order to lower the limit of detection and shorten the preconcentration times), use of different mercury electrodes, extension to other metals (such as $\mathrm{Zn}$ and $\mathrm{Pb}$ ) and other issues will also be considered in forthcoming work related to this promising technique.

Acknowledgements

The authors gratefully acknowledge support of this research by the Spanish Ministry of Education and Science (DGICYT: Project BQU2000-0642), by the European Community under contract EVK1-CT2001-86 and from the "Comissionat d'Universitats i Recerca de la Generalitat de Catalunya".

\section{Appendix A: analytical approach for the measured current}

Let us consider the second step $\left(t>t_{1}\right)$ in the voltammetric implementation (i.e. spherical electrode) of AGNES with detection of the diffusion limited current. The only process to take into account is diffusion of the reduced species according to the equation

$$
\frac{\partial c_{\mathrm{M}^{0}}}{\partial t}=D_{\mathrm{M}^{0}}\left(\frac{\partial^{2} c_{\mathrm{M}^{0}}}{\partial r^{2}}+\frac{2}{r} \frac{\partial c_{\mathrm{M}^{0}}}{\partial r}\right)
$$


In this appendix, let us re-define the origin of time at the beginning of the application of the second step. The initial condition is a flat profile built up in the preconcentration step:

$c_{\mathrm{M}^{0}}(r, 0)=c_{\mathrm{M}^{0}}^{*}$

The boundary conditions are finite volume

$$
\left(\frac{\partial c_{\mathrm{M}^{0}}(r, t)}{\partial r}\right)_{r=0}=0
$$

and the diffusion limited condition:

$$
c_{\mathrm{M}^{0}}\left(r_{0}, t\right)=0
$$

Using Laplace transforms, the solution of the differential equation is shown to be

$$
\bar{c}_{\mathrm{M}^{0}}(r, s)=\frac{c_{\mathrm{M}^{0}}^{*}}{s}-\frac{r_{0} c_{\mathrm{M}^{0}}^{*} \sinh \left(\sqrt{\frac{s}{D_{\mathrm{M}^{0}}} r}\right)}{r s \sinh \left(\sqrt{\frac{s}{D_{\mathrm{M}^{0}}} r_{0}}\right)}
$$

where bar indicates the transformed function and $s$ is the variable in Laplace space.

Backtransformation and differentiation leads to 


$$
I\left(t_{2}\right)=\frac{2000 n F A D_{\mathrm{M}^{0}} c_{\mathrm{M}^{0}}^{*}}{r_{0}} \sum_{m=1}^{\infty} \exp \left[-\frac{m^{2} \pi^{2} t_{2} D_{\mathrm{M}^{0}}}{r_{0}^{2}}\right]=\frac{2000 n F A D_{\mathrm{M}^{0}} c_{\mathrm{M}^{0}}^{*}}{r_{0}} S_{\mathrm{M}^{0}}\left(t_{2}\right)
$$

Using eqns. (5) and (3), we conclude that AGNES proportionality factor for the spherical drop is:

$$
h\left(t_{2}\right)=\frac{2000 n_{e} F A D_{\mathrm{M}^{0}} Y}{r_{0}} S_{\mathrm{M}^{0}}\left(t_{2}\right)
$$

where $S_{\mathrm{M}^{0}}\left(t_{2}\right)$ can be easily computed with the following algorithm:

$$
\begin{array}{ll}
S_{\mathrm{M}^{0}}\left(t_{2}\right) \approx \frac{1}{2}\left(\frac{r_{0}}{\left.\sqrt{\pi t_{2} D_{\mathrm{M}^{0}}}-1\right)} \quad \text { if } \frac{\pi^{2} t_{2} D_{\mathrm{M}^{0}}}{r_{0}^{2}} \leq 0.5\right. \\
S_{\mathrm{M}^{0}}\left(t_{2}\right) \approx \sum_{m=1}^{6} \exp \left[-\frac{m^{2} \pi^{2} t_{2} D_{\mathrm{M}^{0}}}{r_{0}^{2}}\right] & \text { if } \frac{\pi^{2} t_{2} D_{\mathrm{M}^{0}}}{r_{0}^{2}}>0.5
\end{array}
$$

Some selected values of $S_{\mathrm{M}^{0}}\left(t_{2}\right)$ computed with parameters $D_{\mathrm{M}^{0}}=1.6 \times 10^{-9} \mathrm{~m}^{2} \mathrm{~s}^{-1}$ and $r_{0}=1.41 \times 10^{-4} \mathrm{~m}$ are given in the table below

\begin{tabular}{|l|l|l|l|l|}
\hline$t_{2} / \mathrm{s}$ & 0.2 & 0.4 & 1 & 2.5 \\
\hline$S_{\mathrm{M}^{0}}\left(t_{2}\right)$ & 1.7235 & 1.0723 & 0.4944 & 0.1376 \\
& & & & \\
\hline
\end{tabular}


The stripped charge can be computed by integration of eqn. (A-6) up to the given $t_{2}$.

One can check, that if we let $t_{2}$ tend to infinity (i.e. wait until all $\mathrm{M}^{\mathrm{o}}$ is stripped away),

$$
\int_{0}^{\infty} I\left(t_{2}\right) d t_{2}=n F\left(\frac{4}{3} \pi r_{0}^{3}\right) Y c_{\mathrm{M}}^{*}
$$

as expected for a sphere filled at constant concentration $Y c_{\mathrm{M}}^{*}[23]$.

\section{Appendix B: numerical approach to the whole AGNES}

\section{simulation}

We consider here the simulation of AGNES for a reversible couple as the application of

2 different potential steps. For each step, we could compute a corresponding $Y$, so we

indicate this change in $Y$ as $Y(t)$. Diffusion within the drop

$$
\frac{\partial c_{\mathrm{M}^{0}}}{\partial t}=D_{\mathrm{M}^{0}}\left(\frac{\partial^{2} c_{\mathrm{M}^{0}}}{\partial r^{2}}+\frac{2}{r} \frac{\partial c_{\mathrm{M}^{0}}}{\partial r}\right) \quad r<r_{0}
$$

is coupled to diffusion in a restricted domain up to the end of the diffusion layer $\left(r_{1}=\right.$ $r_{0}+1 /\left(1 / \delta-1 / r_{0}\right)$, see eqn. (6) )

$$
\frac{\partial c_{\mathrm{M}}}{\partial t}=D_{\mathrm{M}}\left(\frac{\partial^{2} c_{\mathrm{M}}}{\partial r^{2}}+\frac{2}{r} \frac{\partial c_{\mathrm{M}}}{\partial r}\right) \quad r_{0}<r<r_{1}
$$


via the relationships at the interphase

$$
c_{\mathrm{M}^{0}}^{0}(t)=Y(t) c_{\mathrm{M}}^{0}(t) \quad ; \quad D_{\mathrm{M}^{0}}\left(\frac{\partial c_{\mathrm{M}^{0}}}{\partial r}\right)_{r=r_{0}}=D_{\mathrm{M}}\left(\frac{\partial c_{\mathrm{M}}}{\partial r}\right)_{r=r_{0}} \quad r=r_{0}
$$

together with the finite volume condition $(\mathrm{A}-3)$ and bulk conditions maintained at the edge of the diffusion layer $c_{\mathrm{M}}\left(r_{1}, t\right)=c_{\mathrm{M}}^{*}$.

Via the change

$$
v=c_{\mathrm{M}^{0}} r
$$

eqn. (B-1) can be written as a planar diffusion problem. Analogous transformation can also be done for eqn. (B-2).

The numerical solution of the ensuing coupled partial differential equations was made using a classical finite difference scheme, where the time discretization was carried on by means of an inverse Euler method [44]. 


\section{Appendix C:}

\section{LATIN SYMBOLS}

\begin{tabular}{|c|c|c|c|}
\hline Symbol & Name & Units & Equation \\
\hline$A$ & electrode surface area & $\mathrm{m}^{2}$ & $(\mathrm{~A}-6)$ \\
\hline$c_{\mathrm{M}}(r, t)$ & metal ion concentration (in solution) & $\mathrm{mol} \mathrm{L}^{-1}$ & Fig 1, (B-2) \\
\hline$c_{\mathrm{M}^{0}}(r, t)$ & $\begin{array}{l}\text { reduced metal concentration (in the } \\
\text { amalgam) }\end{array}$ & $\mathrm{mol} \mathrm{L}^{-1}$ & $\begin{array}{l}\text { Fig 1, (A-1), } \\
(\mathrm{B}-1)\end{array}$ \\
\hline$c_{\mathrm{M}^{0}}^{*}$ & $\begin{array}{l}\text { reduced metal concentration for the final flat } \\
\text { profile at } t=t_{1}\end{array}$ & $\mathrm{~mol} \mathrm{~L}^{-1}$ & $(3)$ \\
\hline$c_{\mathrm{M}}^{*}$ & bulk concentration of metal ion $\mathrm{M}$ & $\mathrm{mol} \mathrm{L}^{-1}$ & $(3)$ \\
\hline$c_{\mathrm{M}}^{0}$ & $\begin{array}{l}\text { metal ion concentration at the electrode } \\
\text { surface }\end{array}$ & mol L & $(2)$ \\
\hline$c_{\mathrm{M}^{0}}^{0}$ & $\begin{array}{l}\text { amalgamated metal concentration at the } \\
\text { electrode surface }\left(r=r_{0}\right)\end{array}$ & $\mathrm{mol} \mathrm{L}^{-1}$ & $(2)$ \\
\hline$D_{\mathrm{M}}$ & diffusion coefficient of species $\mathrm{M}$ (metal ion) & $\mathrm{m}^{2} \mathrm{~s}^{-1}$ & $(7)$ \\
\hline
\end{tabular}




\begin{tabular}{|c|c|c|c|}
\hline$D_{\mathrm{M}^{0}}$ & $\begin{array}{l}\text { diffusion coefficient of species } \mathrm{M}^{0} \text { (reduced } \\
\text { metal) }\end{array}$ & $\mathrm{m}^{2} \mathrm{~s}^{-1}$ & (10) \\
\hline$E^{\circ}$ & formal standard potential & V & (3) \\
\hline$E_{1}$ & pre-concentration potential applied along $t \leq t_{1}$ & $\mathrm{~V}$ & (3) \\
\hline$E_{2}$ & stripping potential applied along $t>t_{1}$ & V & Table 1 \\
\hline$E_{\text {peak }}$ & DPP peak potential & V & $(10)$ \\
\hline$\Delta E$ & DPP pulse height & V & $(10)$ \\
\hline$I$ & current & A & $(5)$ \\
\hline$F$ & Faraday constant & $\mathrm{C}$ & (3) \\
\hline$h$ & $\begin{array}{l}\text { proportionality factor between faradaic } \\
\text { current and free metal concentration }\end{array}$ & $\mathrm{A} \mathrm{mol}^{-1} \mathrm{~L}$ & $(5)$ \\
\hline$m$ & dummy index for summation & none & $(\mathrm{A}-6)$ \\
\hline$n$ & number of exchanged electrons & none & (1) \\
\hline$r$ & radial co-ordinate & $\mathrm{m}$ & $(\mathrm{A}-1)$ \\
\hline$r_{0}$ & radius of the spherical electrode & $\mathrm{m}$ & (7) \\
\hline
\end{tabular}




\begin{tabular}{|c|c|c|c|}
\hline$r_{1}$ & $\begin{array}{l}\text { radial co-ordinate of the edge of the diffusion } \\
\text { layer }\end{array}$ & $\mathrm{m}$ & Fig 1 \\
\hline$R$ & gas constant & $\mathrm{J} \mathrm{K}^{-1} \mathrm{~mol}^{-1}$ & (3) \\
\hline$S$ & Laplace variable & $\mathrm{s}^{-1}$ & $(\mathrm{~A}-5)$ \\
\hline$S_{\mathrm{M}^{0}}$ & sum of exponential terms & none & $(\mathrm{A}-6)$ \\
\hline$t$ & time variable & $\mathrm{S}$ & (7) \\
\hline$t_{1}$ & deposition time & $\mathrm{S}$ & (8), Table 1 \\
\hline$t_{2}$ & measuring time (within second step) & $\mathrm{s}$ & $(\mathrm{A}-6)$ \\
\hline$t_{\mathrm{w}}$ & waiting (or rest) time (i.e. no stirring period) & $\mathrm{s}$ & (9), Table 1 \\
\hline$T$ & temperature & K & (3) \\
\hline$v$ & new concentration variable & $\mathrm{M} \mathrm{m}$ & (B-4) \\
\hline$Y$ & concentration gain & none & $(2),(3)$ \\
\hline
\end{tabular}

\section{GREEK SYMBOL}

\begin{tabular}{|l|l|l|l|}
\hline$\delta$ & effective diffusion layer shell thickness & $\mathrm{m}$ & $(7)$ \\
\hline
\end{tabular}


References

[1] J.Buffle, Complexation Reactions in Aquatic Systems. An Analytical Approach., Ellis Horwood Limited, Chichester, 1988.

[2] A.Tessier, J.Buffle and P.G.C.Campbell, in J. Buffle and R. R. DeVitre (Eds.), Chemical and Biological Regulation of Aquatic Systems, Lewis Publishers, Boca Raton, FL, 1994, Chapter 6, p. 197.

[3] J.L.Garces, F.Mas, J.Puy, J.Galceran and J.Salvador, J. Chem. Soc. Faraday Trans. 94 (1998) 2783.

[4] W.H.van Riemsdijk and L.K.Koopal, in J. Buffle and H. P. van Leeuwen (Eds.), Environmental particles, Lewis Publishers, Boca Raton, 1992, Chapter 12,

[5] M.Borkovec, U.Rusch, M.Cernik, G.J.M.Koper and J.C.Westall, Colloids Surf. A 107 (1996) 285.

[6] J.L.Garces, F.Mas, J.Cecilia, E.Companys, J.Galceran, J.Salvador and J.Puy, Phys. Chem. Chem. Phys. 4 (2002) 3764.

[7] R.J.M.Hudson, D.T.Covault and F.M.M.Morel, Mar. Chem. 38 (1992) 209.

[8] P.G.C.Campbell, in A. Tessier and D. R. Turner (Eds.), Metal Speciation and Bioavailability in Aquatic Systems, John Wiley \& Sons, Chichester, 1995, Chapter 2, p. 45 .

[9] P.Buhlmann, E.Pretsch and E.Bakker, Chemical Reviews 98 (1998) 1593.

[10] E.Bakker, P.Buhlmann and E.Pretsch, Chemical Reviews 97 (1997) 3083. 
[11] K.Z.Brainina, E.Y.Neyman, Electroanalytical strypping methods, John Wiley \& Sons, New York, 1993.

[12] H.P.van Leeuwen and R.M.Town, J. Electroanal. Chem. 536 (2002) 129.

[13] M.E.Abdelsalam, G.Denuault and S.Daniele, Anal. Chim. Acta 452 (2002) 65.

[14] E.Companys, J.Puy, M.Torrent, J.Galceran, J.Salvador, J.L.Garces and F.Mas, Electroanal. 15 (2003) 452.

[15] J.P.Bernhard, J.Buffle and N.Parthasarthy, Anal. Chim. Acta 200 (1987) 191.

[16] J.Buffle, J.P.Bernhard and M.L.Tercier, J. Electroanal. Chem. 236 (1987) 67.

[17] J.C.Myland, C.G.Zoski and K.B.Oldham, J. Electroanal. Chem. 193 (1985) 3.

[18] J.E.Anderson, J.C.Myland and K.B.Oldham, J. Electroanal. Chem. 218 (1987) 41.

[19] J.Galceran, J.Salvador, J.Puy, F.Mas, D.Gimenez and M.Esteban, J. Electroanal. Chem. 442 (1998) 151.

[20] R.M.Town, M.L.Tercier, N.Parthasarathy, F.Bujard, S.Rodak, C.Bernard and J.Buffle, Anal. Chim. Acta 302 (1995) 1.

[21] S.P.Kounaves and J.Buffle, J. Electroanal. Chem. 239 (1988) 113.

[22] S.P.Kounaves, W.Deng, P.R.Hallock, G.T.A.Kovacs and C.W.Storment, Anal. Chem. 66 (1994) 418.

[23] J.Schiewe, K.B.Oldham, J.C.Myland, A.M.Bond, V.A.VicenteBeckett and S.Fletcher, Anal. Chem. 69 (1997) 2673. 
[24] J.Koryta, Collect. Czech. Chem. Commun. 24 (1959) 3057.

[25] G.Ginzburg, J. Electroanal. Chem. 90 (1978) 367.

[26] A.Yuchi, H.Wada and G.Nakagawa, Anal. Chim. Acta 149 (1983) 209.

[27] M.Torres, J.M.Diazcruz, C.Arino, B.S.Grabaric, R.Tauler and M.Esteban, Anal. Chim. Acta 371 (1998) 23.

[28] M.Torres, J.M.Diaz-Cruz, C.Arino, B.S.Grabaric and M.Esteban, Electroanal. 11 (1999) 93.

[29] A.I.Vogel, Textbook of Quantitative Chemical Analysis, 5th ed., Longman, London, 1989.

[30] R.M.Rodriguez, E.Brillas and J.A.Garrido, J.Chem.Soc.-Faraday Trans. I 83 (1987) 2813.

[31] K.Tokuda, N.Enomoto, N.Koizumi and H.Matsuda, J. Electroanal. Chem. 159 (1983) 23.

[32] A.Molina, F.Martinez-Ortiz, C.Serna, L.Camacho and J.J.Ruiz, J. Electroanal. Chem. 408 (1996) 33.

[33] F.J.C.Bijl, M.Sluyters-Rehbach and J.H.Sluyters, J. Electroanal. Chem. 435 (1997) 137.

[34] F.Martinez-Ortiz, M.L.Alcaraz, I.Roca and M.Lopez-Tenes, J. Electroanal. Chem. 443 (1998) 243.

[35] F.Garay and V.Solis, J. Electroanal. Chem. 476 (1999) 165.

[36] M.G.Bugarin, A.M.Mota, J.P.Pinheiro and ML.S.Gonçalves, Anal. Chim. Acta 294 (1994) 271.

[37] R.M.Town and H.P.van Leeuwen, J. Electroanal. Chem. 509 (2001) 58.

[38] W.T.de Vries and E.van Dalen, J. Electroanal. Chem. 8 (1964) 366.

[39] H.P.van Leeuwen and R.M.Town, J. Electroanal. Chem. 535 (2002) 1. 
[40] J.P.Pinheiro, A.M.Mota and H.P.van Leeuwen, Colloids Surf. A 151 (1999) 181.

[41] Z.Stojek, B.Stepnik and Z.Kublik, J. Electroanal. Chem. 74 (1976) 277.

[42] A.J.Bard, L.R.Faulkner, Electrochemical Methods, Fundamentals and Applications, Wiley, New York, 1980.

[43] Allison,J.D., $\quad$ Brown,D.S., and Novo-Gradac,K.J. MINTEQA2/PRODEFA2, A geochemical assessment model for environmental systems: version 3.0 user's manual. Washington, DC.7. U.S. Environmental Protection Agency, Office of Research and Development. 1991; EPA 600/3-91/021; U.S.

[44] Ames,W.F. Numerical methods for partial differential equations. New York. Academic Press. 1992;

\section{Table}

\begin{tabular}{|l|l|l|l|}
\hline time & $0<t<t_{1}-t_{\mathrm{w}}$ & $t_{1}-t_{\mathrm{w}}<t<t_{1}$ & $t_{1}<t<t_{1}+t_{2}$ \\
\hline applied potential & $E_{1}$ & $E_{1}$ & $E_{2}$ \\
\hline stirring & on & off & off \\
\hline
\end{tabular}

Table 1: Potential and stirring program of a simple implementation of AGNES. 


\section{Figures}

1a)

$$
t=t_{1}
$$

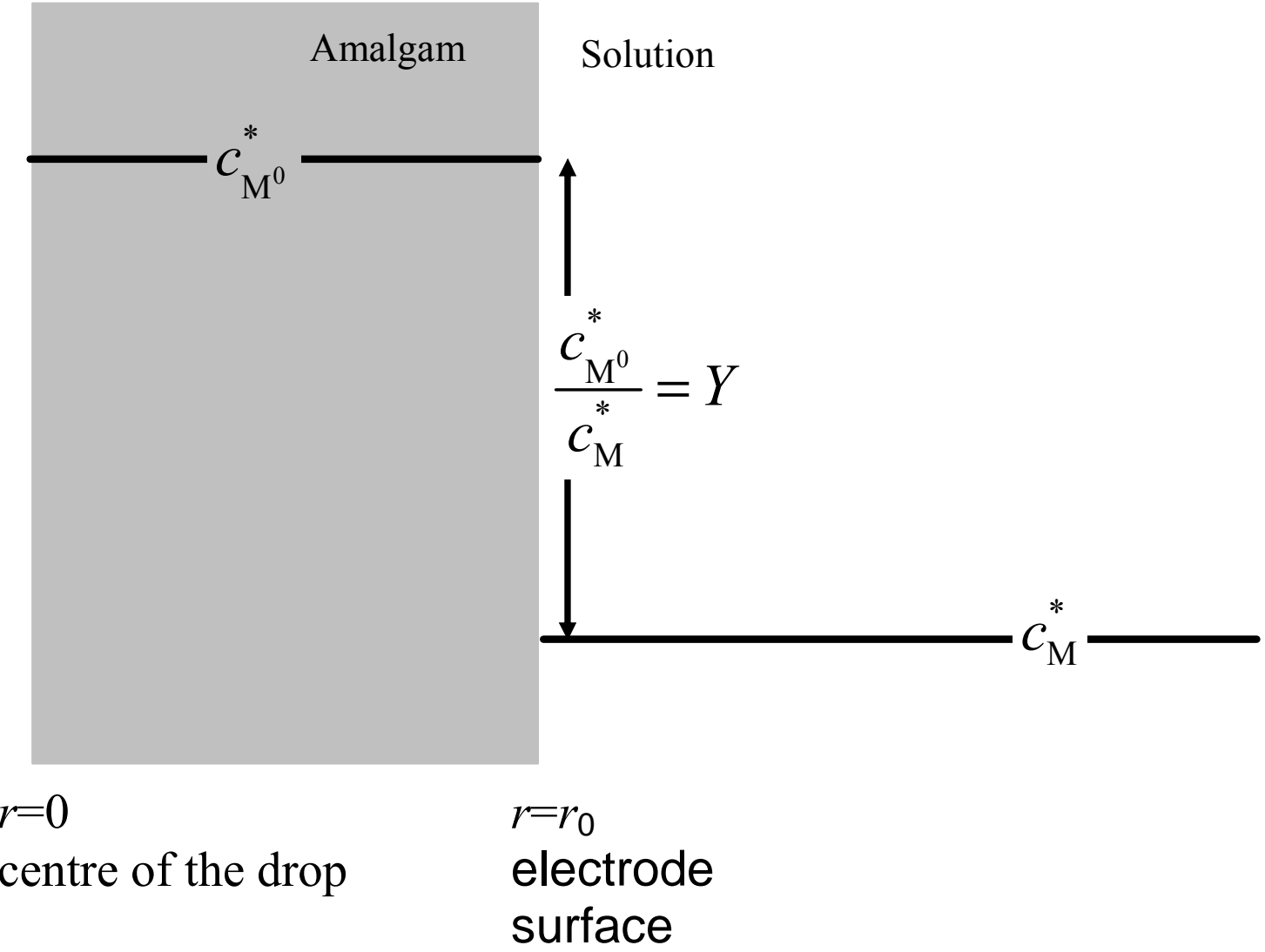


1b)

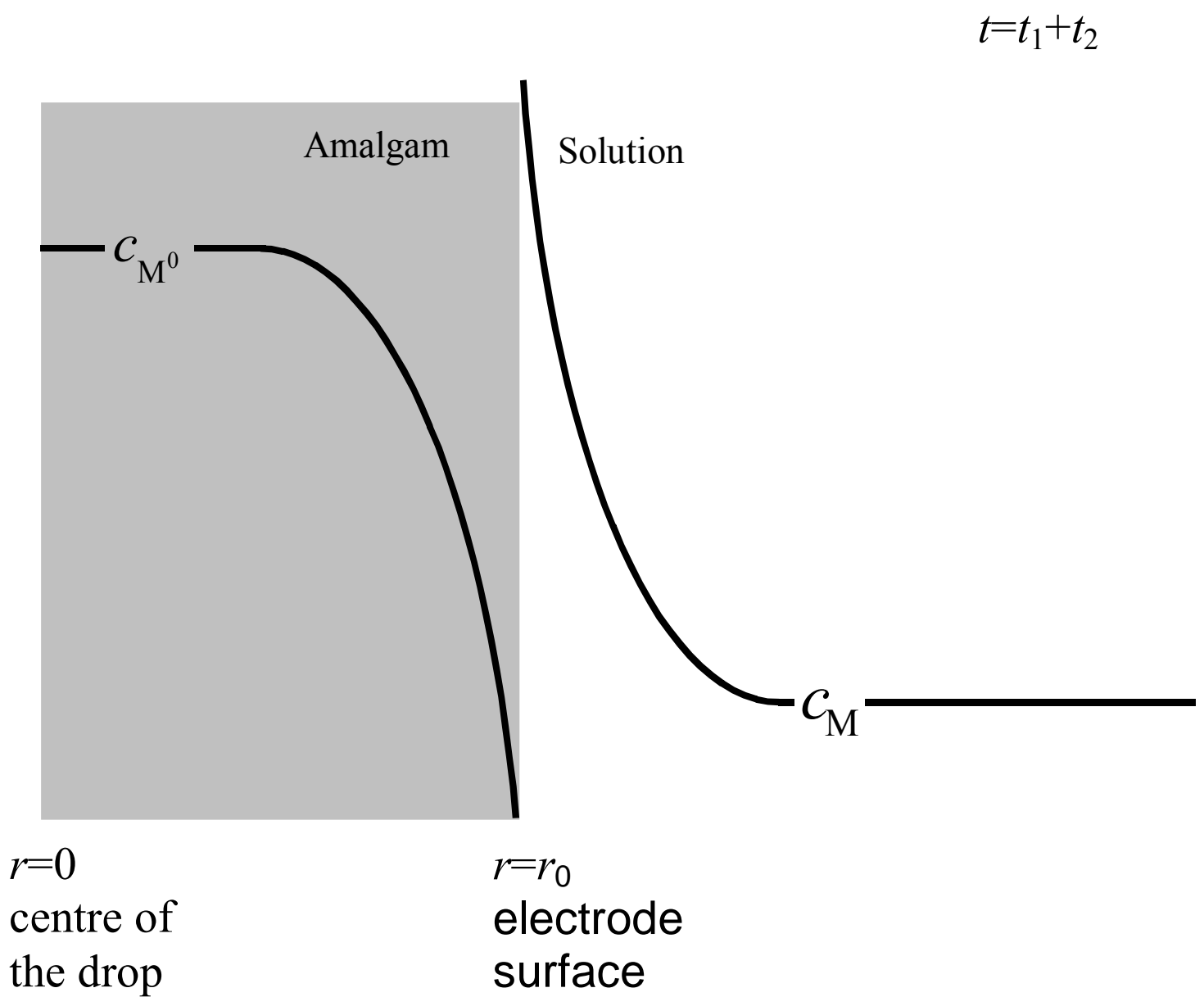

Fig 1 : Outline of concentration profiles inside (left, in the grey region) and outside the mercury electrode. a) Profiles at the end of the first step $\left(t=t_{1}\right)$ exhibiting no concentration gradient in any phase and a fixed concentration ratio $Y$ (due to the Nernstian equilibrium) at the interphase. b) Profiles after $t_{2} \mathrm{~s}$ of the second step have elapsed, when the response current is measured. For clarity purposes the notes at the feet refer to the voltammetric implementation of AGNES with a drop of radius $r_{0}$. 
Published in Journal of Electroanalytical Chemistry 2004, vol 566, p 95-109

DOI: 10.1016/j.jelechem.2003.11.017

2a)

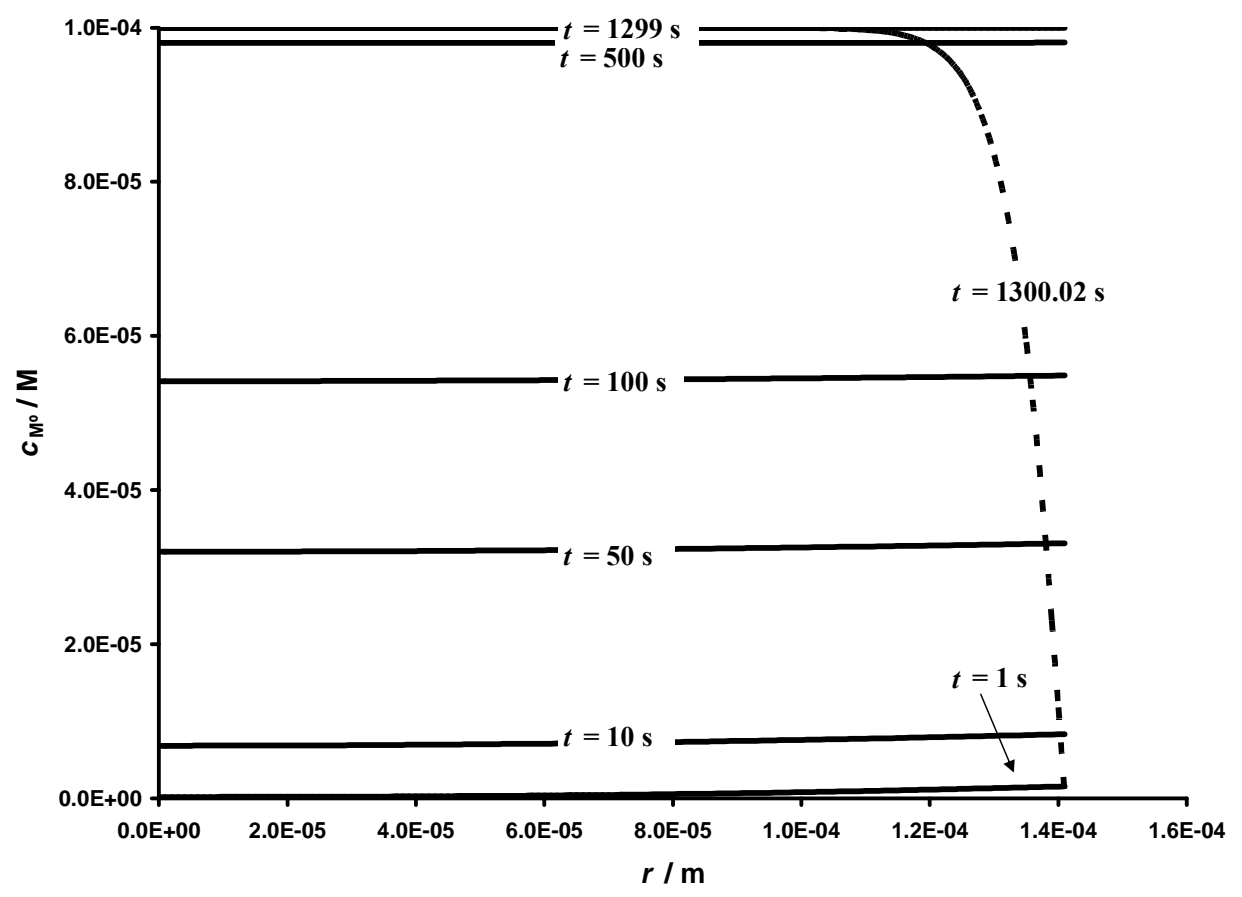

2b)

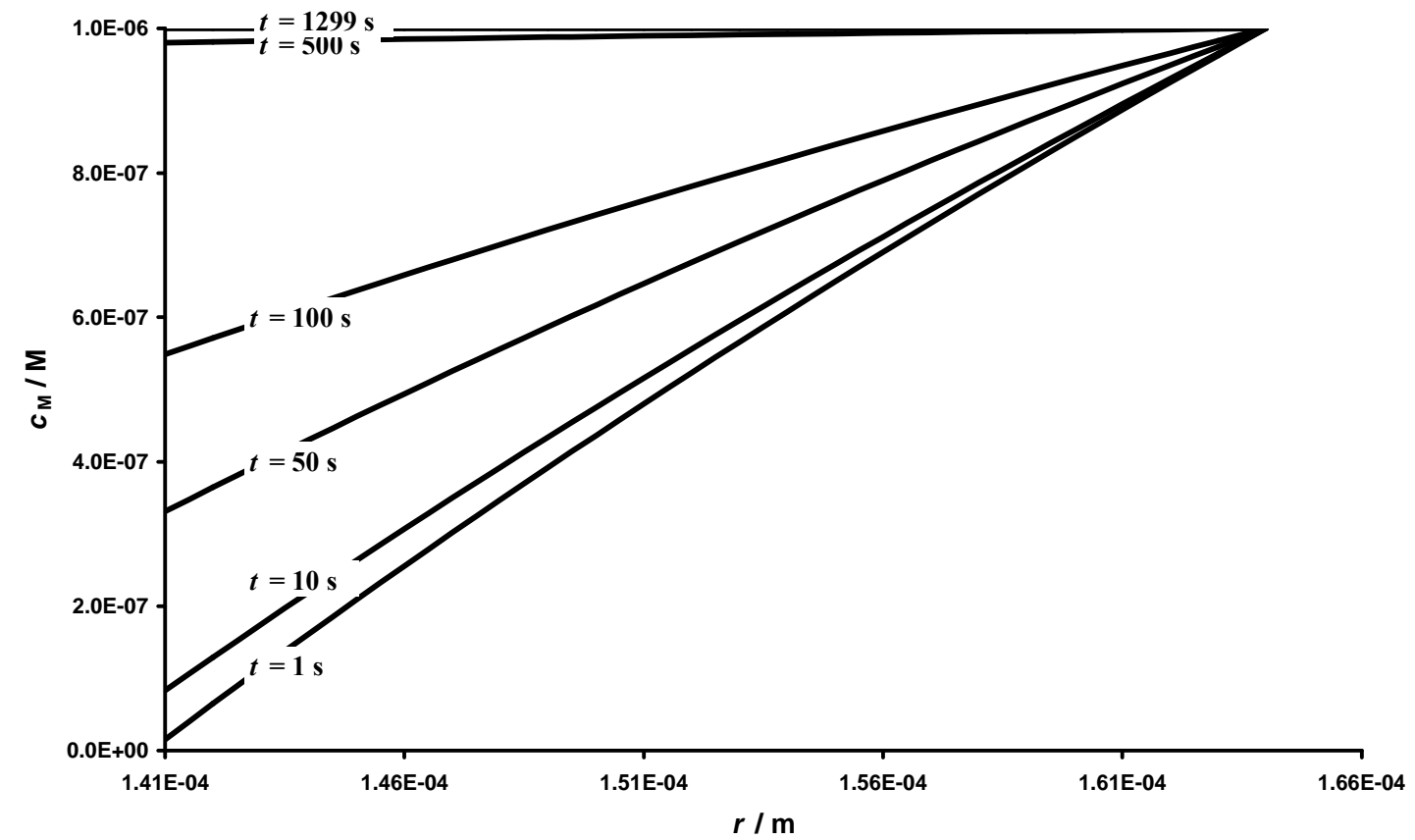


Fig 2: Evolution of the concentration profiles of $c_{\mathrm{M}^{0}}$ (sub-figure a, inside the drop) and $c_{\mathrm{M}}$ (sub-figure $\mathrm{b}$, outside the drop) according to the numerical model for a spherical electrode detailed in appendix B. For illustration, only one $c_{\mathrm{M}^{0}}$-profile for $t>t_{1}$ (corresponding to $t=1300.02 \mathrm{~s}$, i.e. $t_{2}=0.02$, and drawn in dashed line) has been included in sub-figure a. Parameters: $c_{\mathrm{M}}^{*}=10^{-6} \mathrm{M} ; D_{\mathrm{M}^{0}}=1.6 \times 10^{-9} \mathrm{~m}^{2} \mathrm{~s}^{-1} ; D_{\mathrm{M}}=7.3 \times 10^{-10} \mathrm{~m}^{2} \mathrm{~s}^{-1} ; r_{0}=$ $1.41 \times 10^{-4} \mathrm{~m} ; t_{1}=1300 \mathrm{~s} ; Y=100$ (corresponding to $E_{1}-E^{\mathrm{o}}, \approx-59 \mathrm{mV}$ ) and $r_{1}=1.64 \times 10^{-4}$ $\mathrm{m}\left(\delta 22 \times 10^{-5} \mathrm{~m}\right)$ 


\section{a fixed $t<t_{1}$}

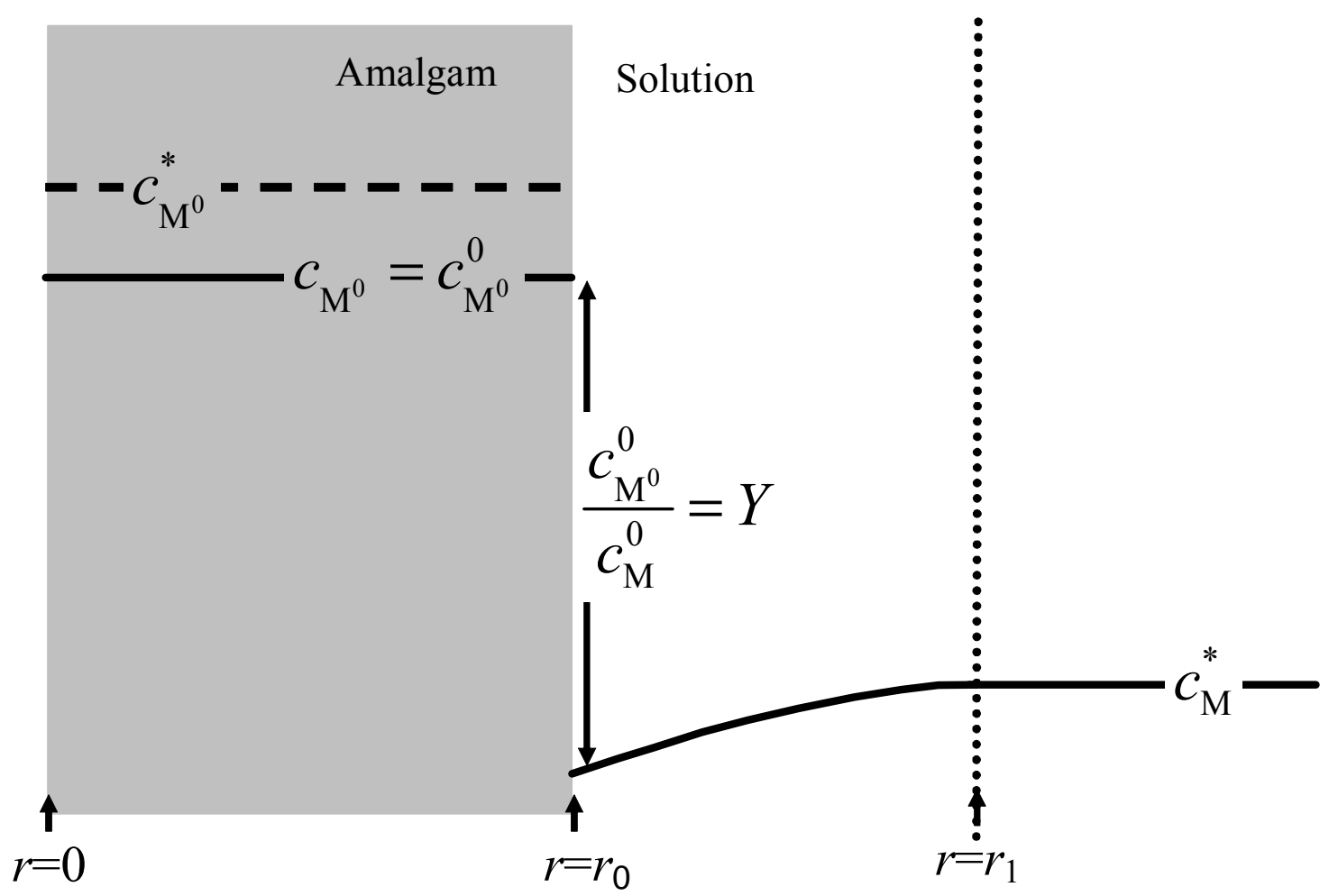

Fig 3 : Outline of a simple model for a voltammetric implementation of AGNES.

Diffusion is fast in comparison with the filling up process, so that at any fixed $t<t_{1}$ one can assume a flat concentration profile inside the drop and a steady-state concentration profile in the solution up to a certain radial position $r_{1}=r_{0}+1 /\left(1 / \delta-1 / r_{0}\right)$ where bulk conditions are restored due to stirring. The dashed horizontal line indicates the final flat profile for $c_{\mathrm{M}^{0}}$. 


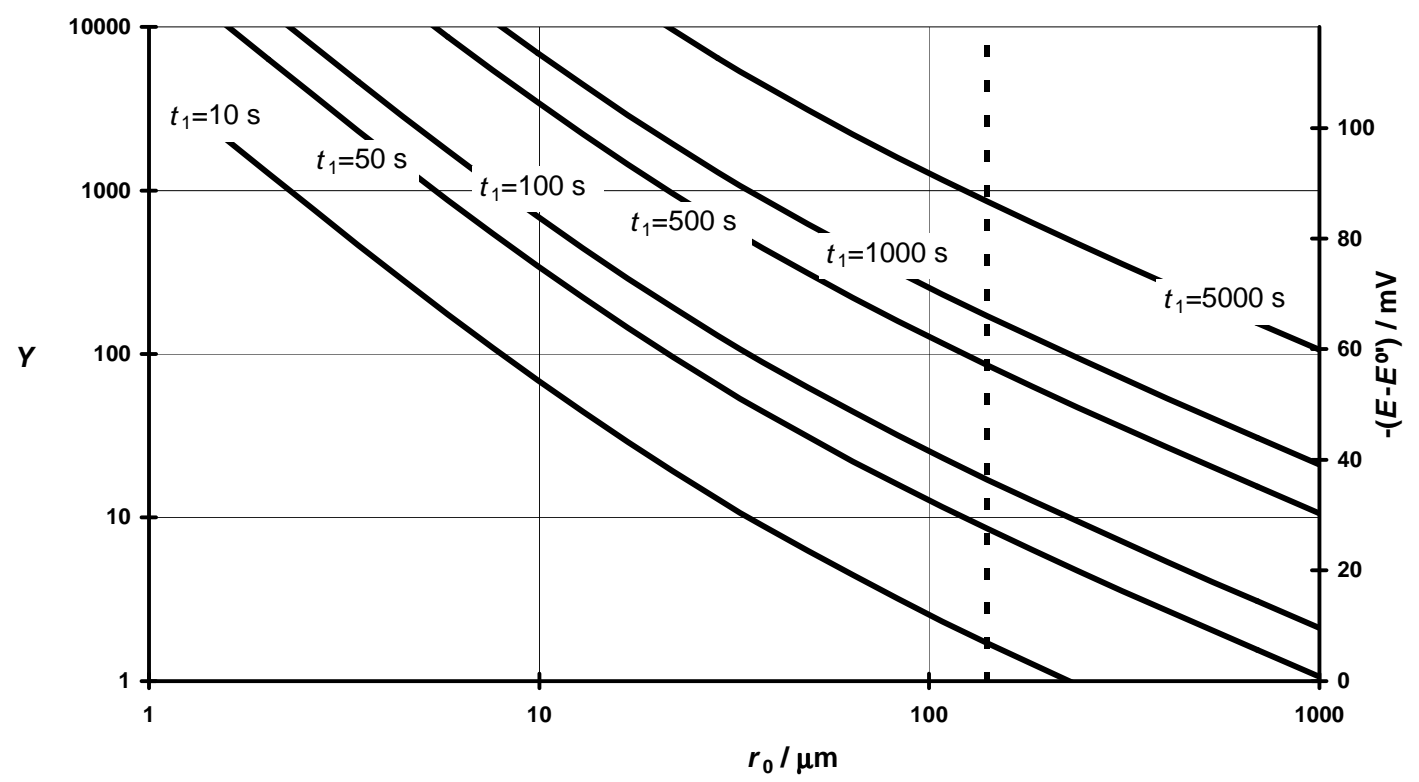

Fig 4 Contour plot of required times $t_{1}$ to reach $99 \%$ of the equilibrium value $\left(c_{\mathrm{M}^{0}}^{*}=0.99 Y c_{\mathrm{M}}^{*}\right.$, according to the simple spherical model given by eqn. (8)) in terms of the preconcentration factor $Y$ and the radius $r_{0}$. Each iso-curve connects couples of $\left(r_{0}\right.$, $Y)$ requiring the $t_{1}$-value indicated in the label. The value of the electrode radius estimated for the experiments in this work is indicated as a vertical dashed line. Parameters: $c_{\mathrm{M}}^{*}=1.72 \times 10^{-5} \mathrm{M} ; D_{\mathrm{M}}=7.3 \times 10^{-10} \mathrm{~m}^{2} \mathrm{~s}^{-1} ; r_{0}=1.41 \times 10^{-4} \mathrm{~m}$ and $r_{1}-r_{0}=2.3 \times 10^{-5}$ m (used to compute $\delta$ with eqn.(6)). The right hand side ordinate axis indicates the required potential difference $-\left(E_{1}-E^{\mathrm{o}}\right)$ necessary to obtain the $Y$-value indicated in the left hand side ordinate axis for a divalent metal $(n=2)$ according to the relationship $-\left(E_{1}-E^{\mathrm{o} \prime}\right)=\frac{R T}{n F} \ln Y$ which follows from eqn. (3). 


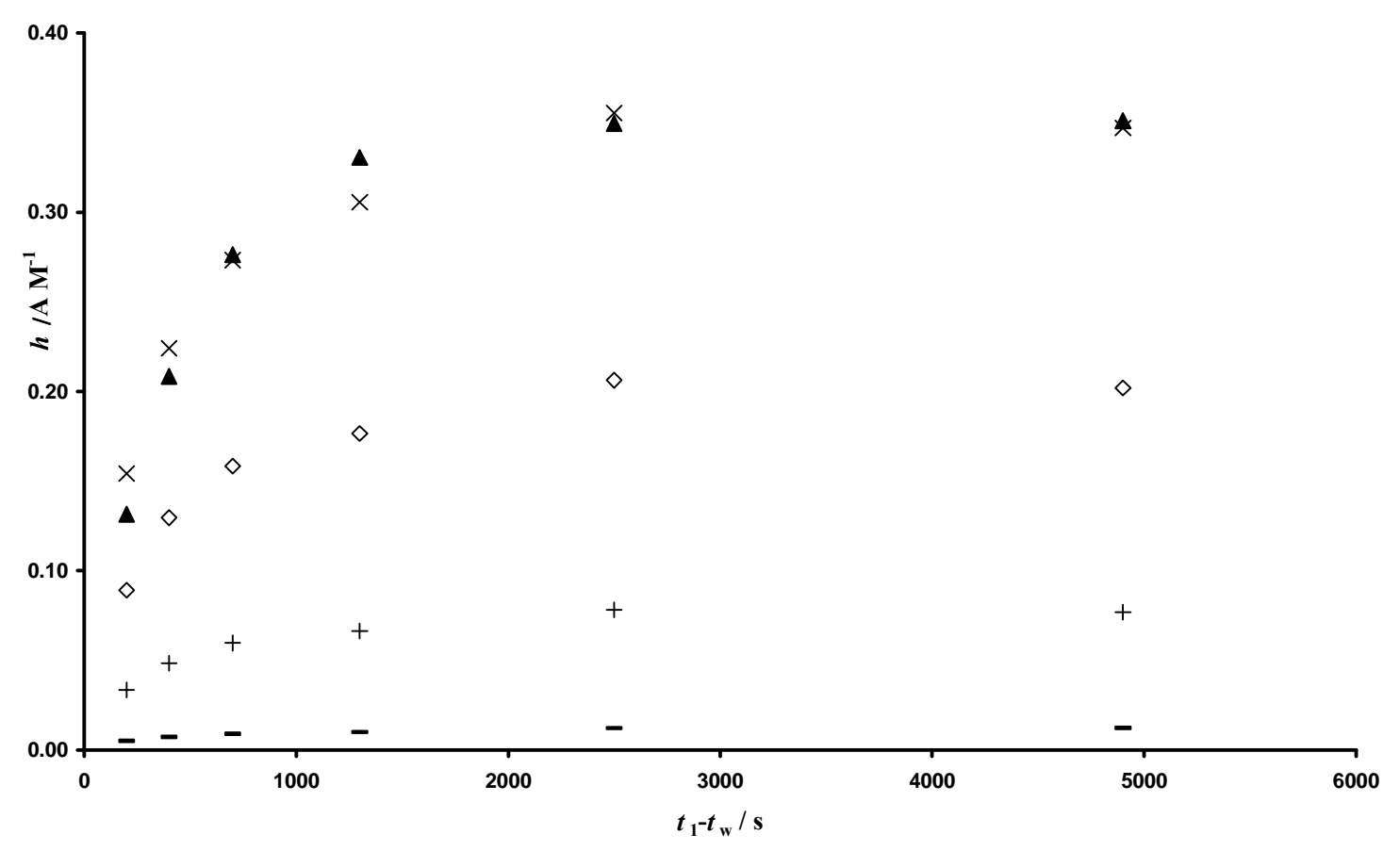

Fig 5: Plot of the proportionality factor $h=I / c_{\mathrm{M}}^{*}$ for various stirred preconcentration times $t_{1}-t_{\mathrm{w}}$. Notice that for shorter times (e.g. $t_{1}-t_{\mathrm{w}}<2000 \mathrm{~s}$ ), the system did not reach absence of gradients due to the relatively high value imposed to $Y$ via $E_{1}$. Total Cd concentration $1.02 \times 10^{-6} \mathrm{M}$, free metal concentration computed with MINTEQ= $9.42 \times 10^{-7}$ M. Markers for experimental data: $\times, t_{2}=0.2 \mathrm{~s} ; \diamond$ (diamond), $t_{2}=0.4 \mathrm{~s} ;+$, $t_{2}=1.0 \mathrm{~s} ;-, t_{2}=2.5 \mathrm{~s}$. Settings: $E_{1}=-0.670 \mathrm{~V}, t_{\mathrm{w}}=100 \mathrm{~s}$. The simple model described by eqn. (9) (solid triangle markers), with parameters $t_{2}=0.2 \mathrm{~s}, t_{\mathrm{w}}=100 \mathrm{~s}, D_{\mathrm{M}^{0}}=1.6 \times 10^{-9} \mathrm{~m}^{2} \mathrm{~s}^{-}$ ${ }^{1} ; r_{0}=1.41 \times 10^{-4} \mathrm{~m} ; Y=292$ (computed from the applied potentials and DPP peak using eqn. (10)) and $\delta=2.47 \times 10^{-5} \mathrm{~m}$ approximately follows the filling up of the drop. 


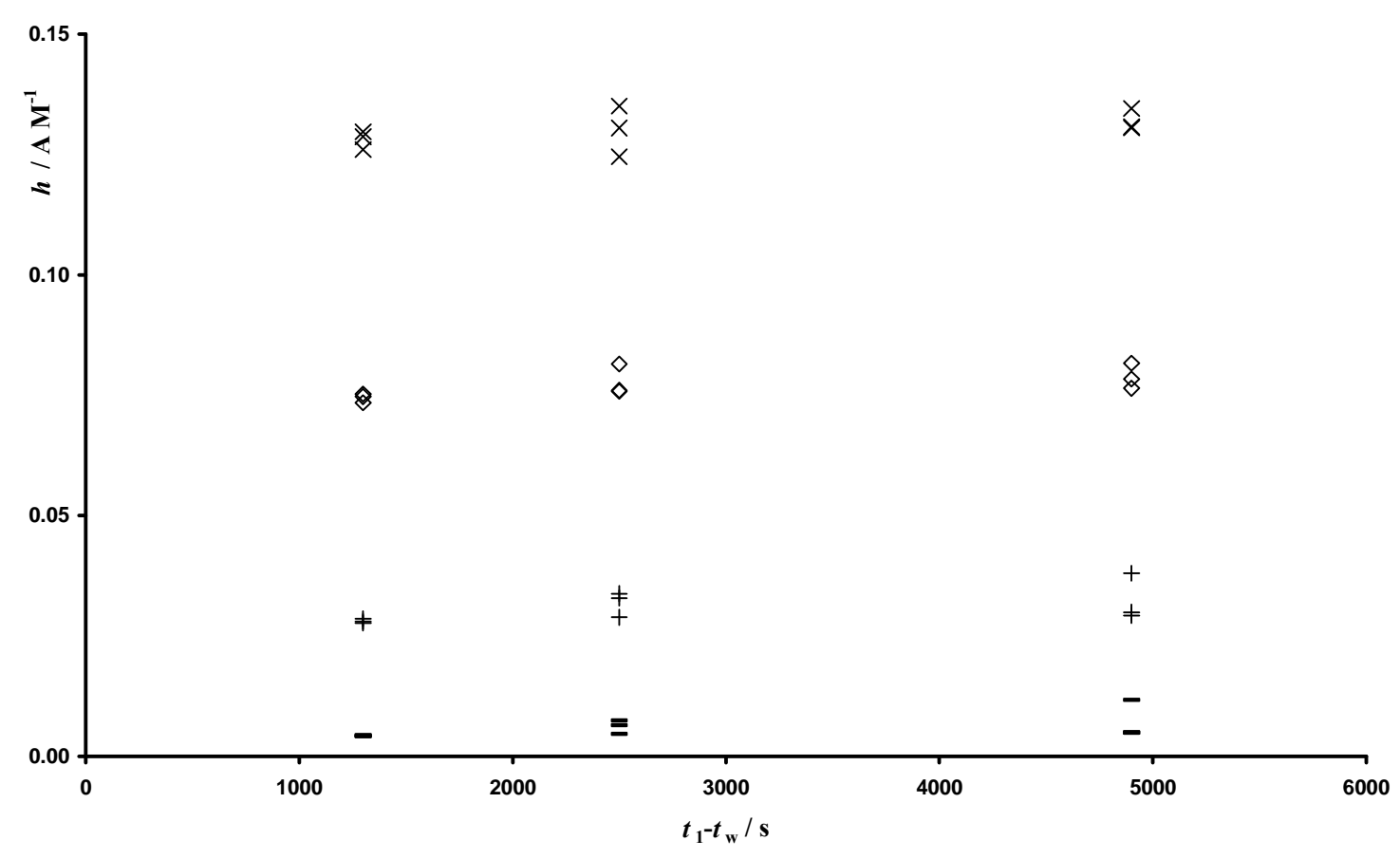

Fig 6: The constancy shown by $h$ at different long times for $t_{1}-t_{\mathrm{w}}$ (three replicates for each stirring time are included in the plot) indicates the suitability of the selected parameters for this experimental system and set-up. Total Cd concentration $=1.02 \times 10^{-6}$ $\mathrm{M}$, free metal concentration computed with $\mathrm{MINTEQ}=9.42 \times 10^{-7} \mathrm{M} . E_{1}=-0.660 \mathrm{~V}$ corresponded to $Y=124$. Markers for different $t_{2}$ as in figure 5. From these kind of experiments we drew the practical conclusion of using $t_{1}=1400 \mathrm{~s}$, while keeping $t_{\mathrm{W}}=100$ S. 


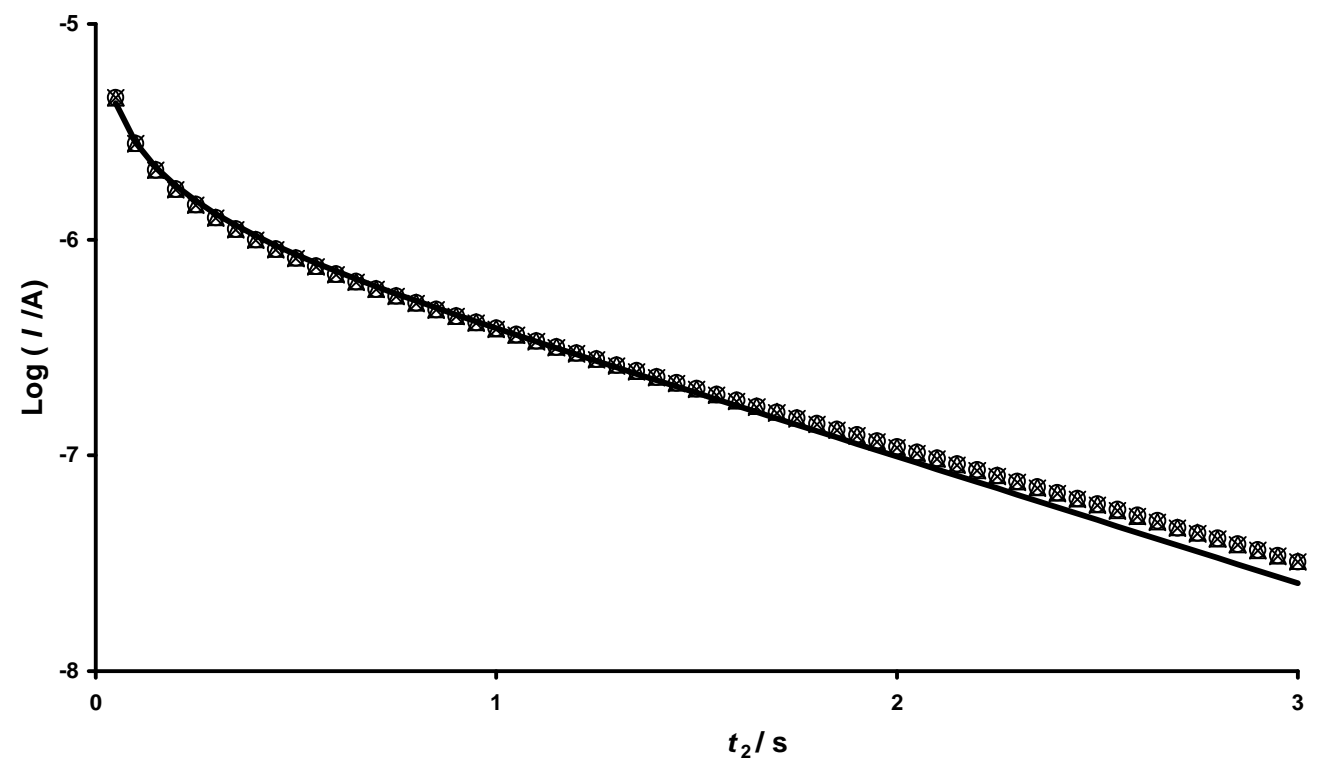

Fig 7: Comparison between the theoretical current for the perfectly spherical drop according to eqn. (A-6) (solid line) and the experimental current decay measured in 3 replicates (markers) along the stripping step $\left(t>t_{1}\right)$ for a solution $1.78 \times 10^{-5} \mathrm{M}$ in free $\mathrm{Cd}$ concentration. Experimental settings: $t_{1}=1400 \mathrm{~s}, t_{\mathrm{w}}=100 \mathrm{~s}, E_{1}=-0.699 \mathrm{~V}, \quad Y=99$. Parameters used in eqn. (A-6): $D_{\mathrm{M}^{0}}=1.6 \times 10^{-9} \mathrm{~m}^{2} \mathrm{~s}^{-1} ; r_{0}=1.08 \times 10^{-4} \mathrm{~m}$ (computed with eqn. (A-9) from the measured charge) and $Y=99$ (computed from the applied potentials and DPP peak using eqn. (10)) . 


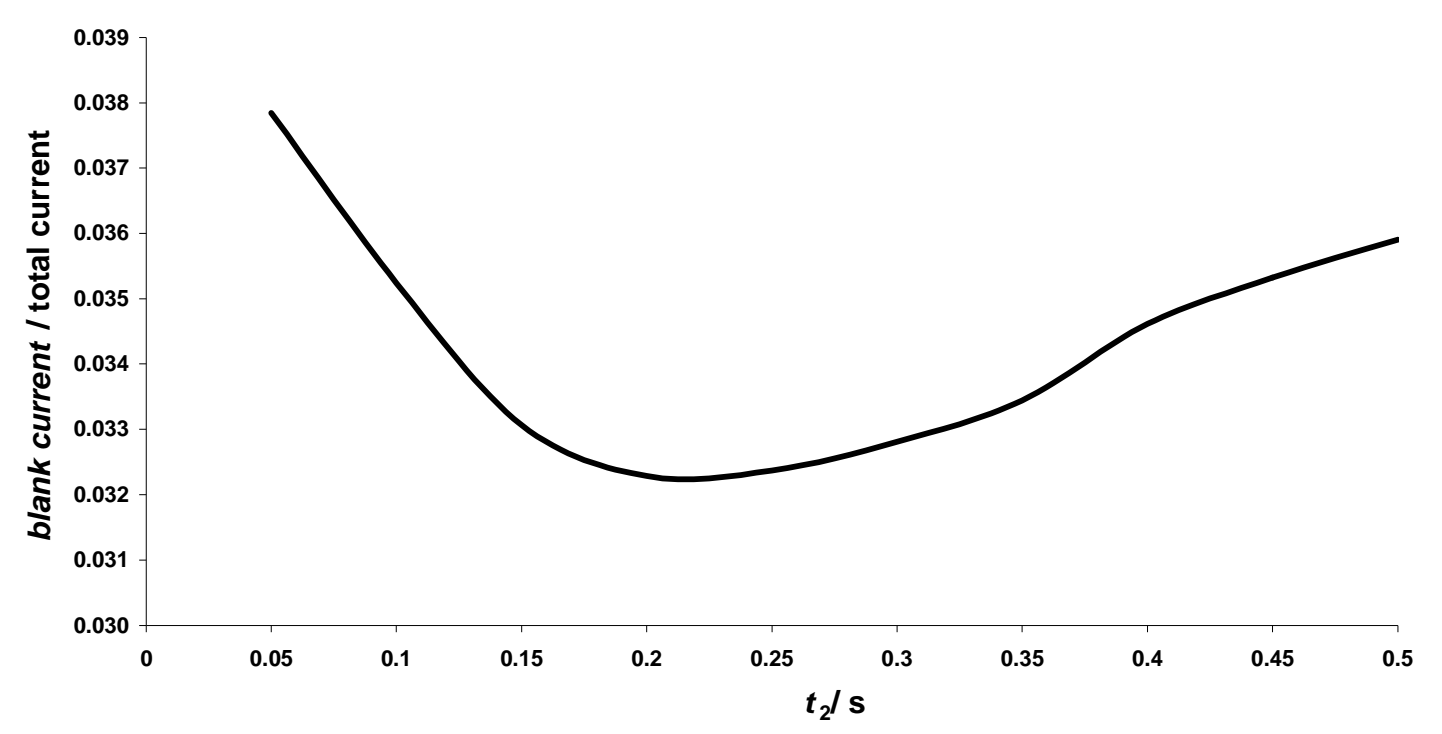

Fig 8: Ratio of blank (just $\mathrm{KNO}_{3}$ in the solution) and total (with total Cd concentration

$1.02 \times 10^{-6} \mathrm{M}$ ) currents showing the convenience of using $t_{2}=0.2 \mathrm{~s}$ as suitable measuring time. Settings: $t_{1}=2600 \mathrm{~s}, t_{\mathrm{w}}=100 \mathrm{~s}, E_{1}=-0.660 \mathrm{~V}, Y=145$. 


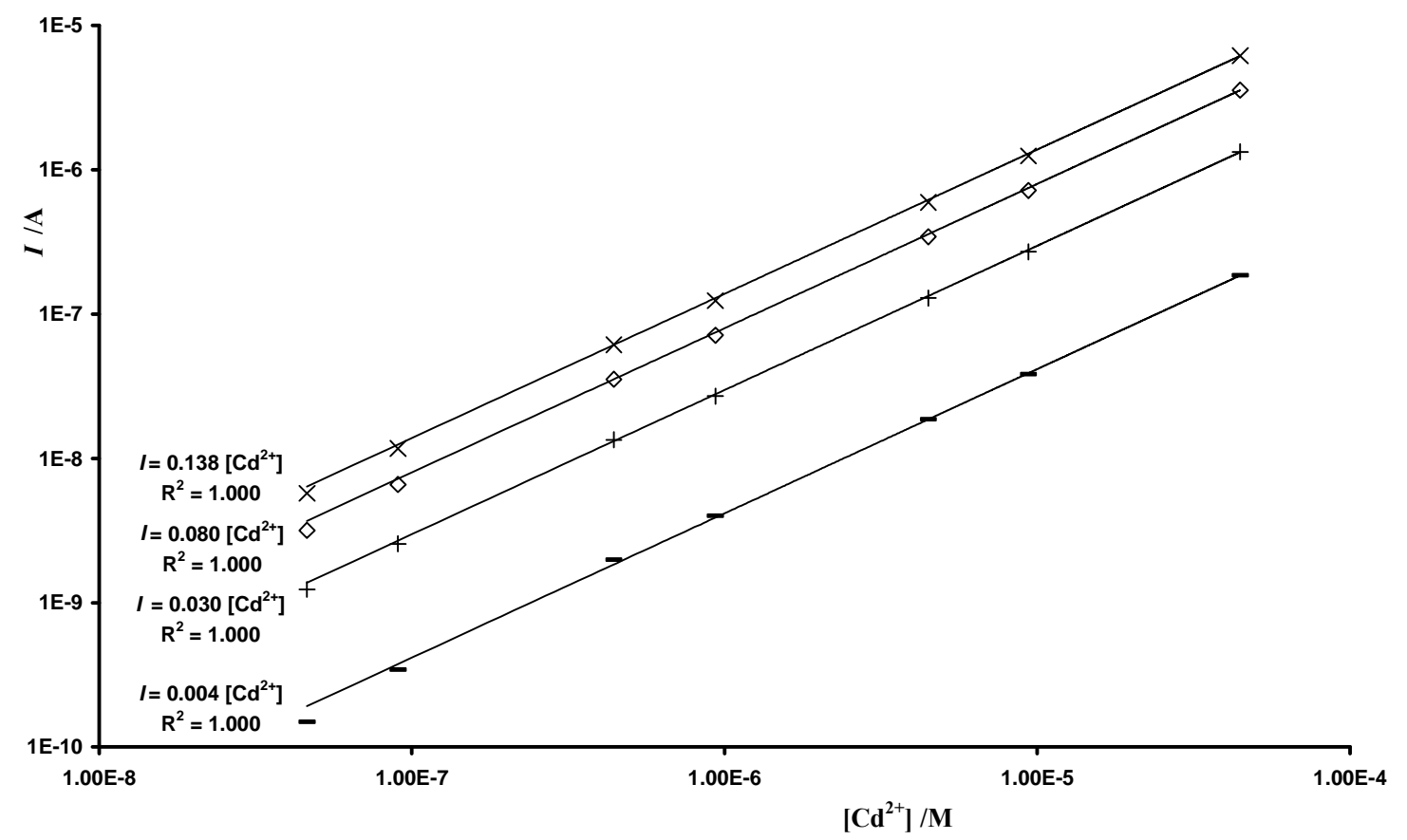

Fig 9: Plot of I versus $c_{\mathrm{M}}^{*}$ for different measuring times $\left(t_{2}\right)$, with markers as in previous

figures. Settings: $t_{1}=950 \mathrm{~s}, t_{\mathrm{w}}=100 \mathrm{~s}, E_{1}=-0.650 \mathrm{~V}$. 


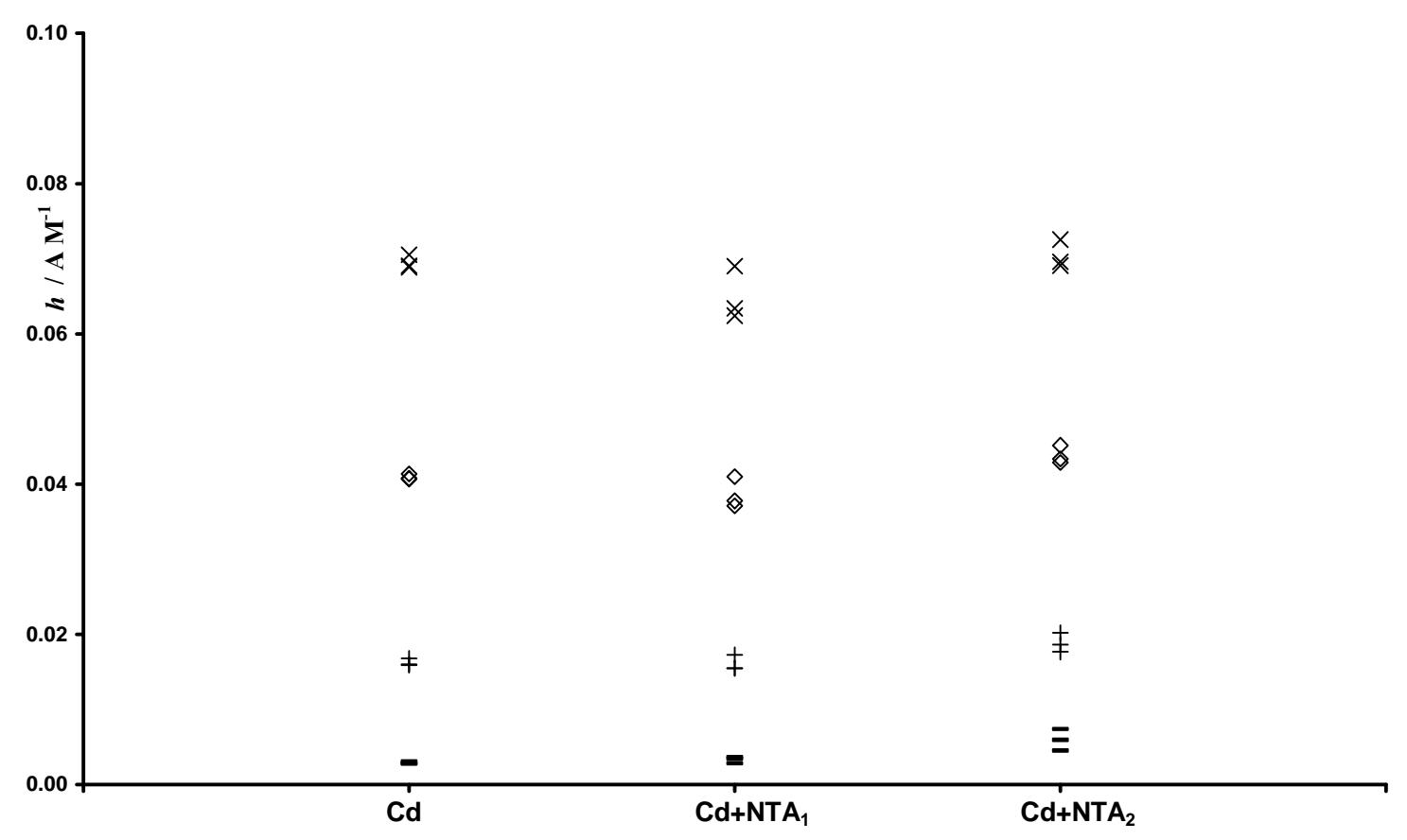

Fig 10: A practically constant value of $h$ found in 3 replicates of each mixture of $\mathrm{Cd}$ and NTA indicates that AGNES senses the free metal concentration (as computed by MINTEQ). $\mathrm{pH}$, total Cd and NTA concentrations at each point: only Cd solution (5.500, $\left.1.9410^{-5} \mathrm{M}, 0 \mathrm{M}\right), \mathrm{Cd}+\mathrm{NTA}_{1}$ solution $\left(4.822,1.9410^{-5} \mathrm{M}, 1.02 \times 10^{-4} \mathrm{M}\right), \mathrm{Cd}+\mathrm{NTA}_{2}$ solution $\left(4.902,1.8910^{-5} \mathrm{M}, 1.21 \times 10^{-3} \mathrm{M}\right)$. Experimental settings $t_{1}=1400 \mathrm{~s}, t_{\mathrm{w}}=100 \mathrm{~s}$, $E_{1}=-0.600 \mathrm{~V}, Y=40.5$. 


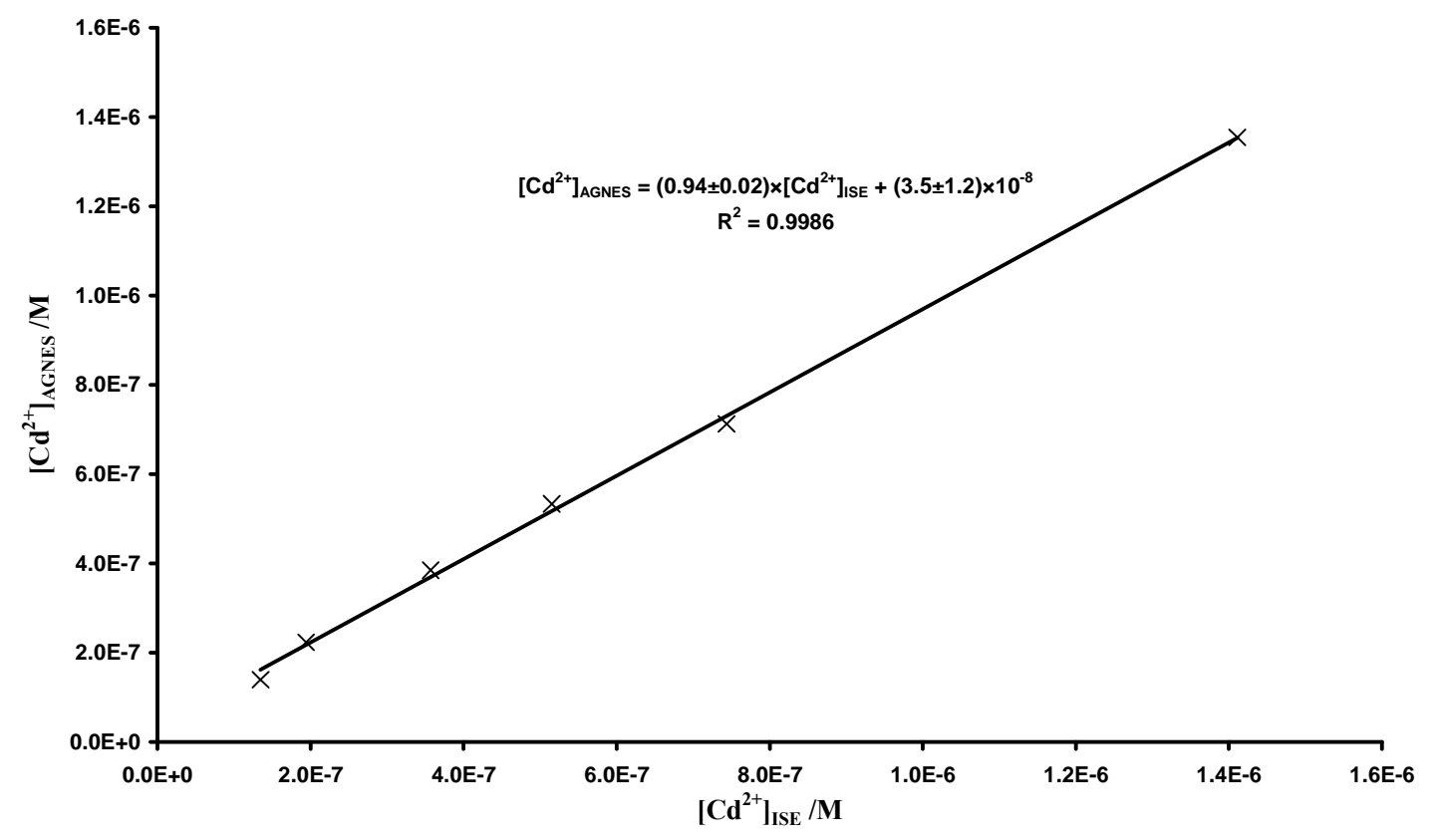

Fig 11: Correlation between free $\mathrm{Cd}^{2+}$ determined by ISE and AGNES at $t_{2}=0.2 \mathrm{~s}$ along a titration with NTA of a solution containing $1.92 \times 10^{-5} \mathrm{M} \mathrm{Cd}$. Experimental settings: $t_{1}=1400 \mathrm{~s}, t_{\mathrm{w}}=100 \mathrm{~s}, E_{1}=-0.699 \mathrm{~V}, Y=99$. 


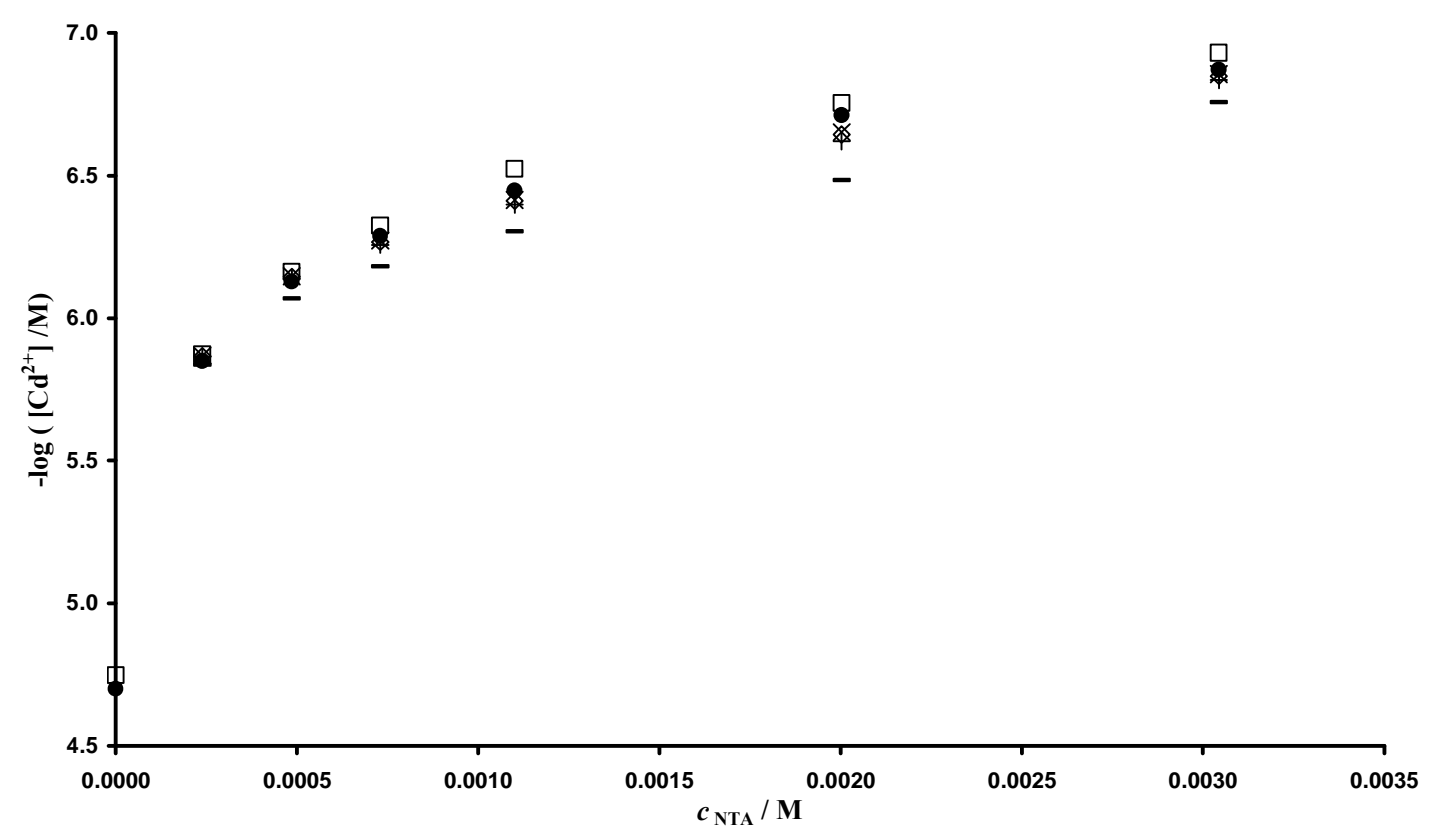

Fig 12: Comparison of free Cd concentration obtained for the titration of the previous figure as determined by AGNES (with markers for different measuring times as in figure 5), Cd-ISE (marker filled bullet •) and predicted by MINTEQ (marker square $\square$ ). 